SINAI Journal of Applied Sciences 9 (1) 2020 105-116

\begin{tabular}{lcc} 
Available online at www.sinjas.journals.ekb.eg & $\overline{\text { ARU-EGYPT }}$ \\
\hline SCREENED BY SINAI Journal of Applied Sciences
\end{tabular}

\title{
AN ECONOMIC STUDY OF THE FISH PRODUCTION SYSTEM IN EGYPT
}

\author{
Ashraf M.A. El-Nagar ${ }^{*}$, M.A. El-Sayed ${ }^{2}$, R.I.M. Radwan ${ }^{2}$ and R.M. Hefny ${ }^{2}$ \\ 1. Agric. Quaran. in Ismailia, Min. Agric., Egypt. \\ 2. Dept. Econ. and Rural Develop., Fac. Environ. Agric. Sci., Arish Univ., Egypt.
}

\section{ARTICLE INFO}

Article history:

Received: $05 / 01 / 2020$

Revised: 30/03/2020

Accepted: 27/04/2020

Available online: 02/05/2020

Keywords:

Economics production,

fish sector,

bony fish,

cartilaginous fish,

fishing boats.

\begin{abstract}
Egypt has large areas of water fisheries, in addition to many lakes of different characteristics, and the Nile River, but these sources are not exploited sufficiently. Despite the increase in the amount of fish production in Egypt from about 1.45 million tons in 2013 to about $1.82 \%$ million tons in 2017 , with an increase of $25.0 \%$, the price of fish has increased from about LE $13.5 / \mathrm{kg}$ in 2013 to about LE $24 / \mathrm{kg}$ in 2017 , Which indicates that the quantities produced of fish do not meet the increasing consumer needs, and the research aims to identify the current status of fish production and ingredients. The basic characteristics of this activity in Egypt through studying the current status of fish production and value and studying the current state of development of fish production according to fisheries, fish groups and the basic ingredients (production requirements) of fish production activity in Egypt. The total fish production amounted to a minimum of about 724.3 thousand tons in 2000, while its maximum amount reached about 1822.8 thousand tons in 2017 and the average price per ton has reached its limit The minimum is about LE 7.7 thousand, while the maximum amount reached about LE24 thousand As for the evolution of the amount of fish production in Egypt according to fisheries, fish production in marine waters during the period (2000-2017) reached a minimum of 102.9 thousand tons, while its maximum reached 136.2 thousand tons, while fish production from lakes has reached a minimum of about 144 thousand tons, while its maximum reached about 195.4 thousand tons, and the minimum amount of freshwater fish production reached about 66.1 thousand tons, while its maximum reached about 120.9 thousand tons. As for the evolution of production from fish farming reached a minimum of about 340.1 thousand tons in the year 2000, while its maximum amount reached about 1451.8 thousand tons. As for the development of fish production according to fish groups during the period (2000-2017), it was found that fish production from bony fish has reached a minimum of about 668.6 thousand tons, while its maximum reached about 1772.9 thousand tons, while fish production from cartilaginous fish has the minimum reached about 0.6 thousand tons, while the maximum reached about 3.6 thousand tons, while the fish production from crustaceans has reached a minimum of about 11.8 thousand tons, while the maximum amount reached about 25.8 thousand tons. As for With regard to fish production from mollusks, the minimum reached about 1.9 thousand tons, while the maximum amount reached about 4.8 thousand tons and it was found that fish production of other varieties has reached a minimum of about 14.1 thousand tons, while its maximum amount reached about 35 thousand tons. Regarding the basic constituents (production requirements) within the fish production activity during the period (2000-2017), the number of motorized fishing boats reached the lowest value of them about 3.8 thousand boats, while its maximum limit reached about 5.1 thousand boats. As for the number of non-motorized fishing boats, the lowest value reached about 24.2 thousand boats, while the maximum limit reached about 42.2 thousand boats. And it also turned out that the minimum number of fishers reached about 22.3 thousand fishermen, while it reached its limit maximum is about 60.4 thousand fishermen in 2000 . As for the number of local societies working in fishing activity, it was found that the minimum reached about 80 associations, while the maximum number reached about 89 associations. While the minimum level for fuel and oils reached about LE 9.2 million in one year, while the maximum amount reached about LE 89.5 million.
\end{abstract}

\footnotetext{
* Corresponding author: E-mail address: ashraf.alnaggar74@gmail.com https://doi.org/10.21608/SINJAS.2020.86430

(C) 2020 SINAI Journal of Applied Sciences. Published by Fac. Environ. Agric. Sci., Arish Univ. All rights reserved.
} 
العـام لتطـور الكميـات المنتجـة مـن الأسماك و المقومـات الأساسية لهذا النشاط ووصو لا لتحقيق الأهداف المطلوبة.

\section{مصادر جمع البيانات}

اعتمدت الدر اسة على بعض البيانات الإحصائية الثانوية

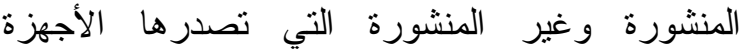

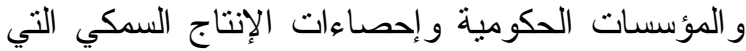

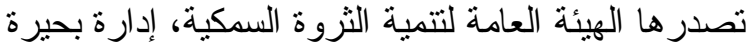

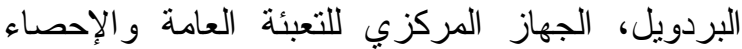
بالإضافة إلى بعض الإد الدرأسات العلمية ذات الصلة الصائة

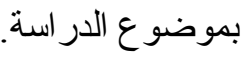

\section{النتائج والمناقشة}

\section{تطور كمية وقيمة إنتاج الأسماك فى مصر}

يوضح جدول ا نطور كمبة وقيمة إنتاج الأسماك

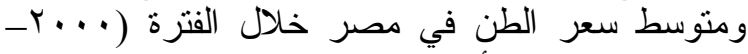

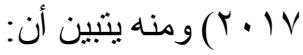

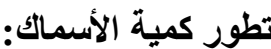

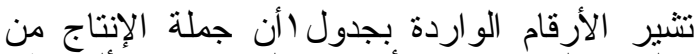

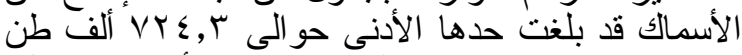

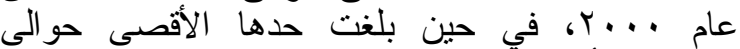

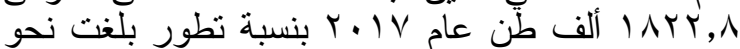

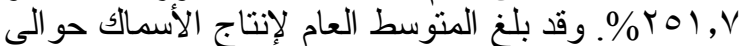

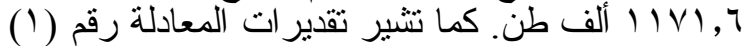
بجدول r معادلة الاتجاه الزمنى العام لتطور كمية إنتاج

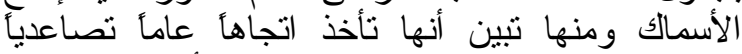

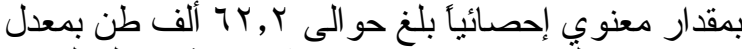

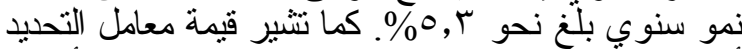

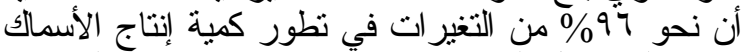

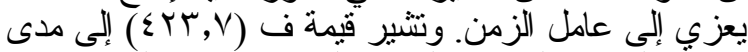
مطابقة النموذج المستخدم لطبيعة الييانات موضع القياس.

\section{تطور قيمة الإنتاج السمكي}

يوضح جدول ا قيمة إنتاج الأسماك خلال الفترة

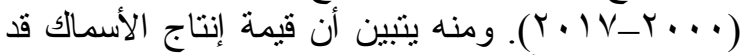

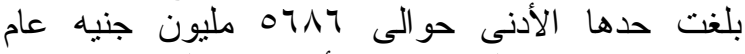

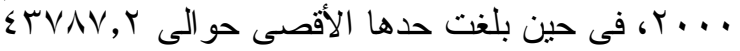

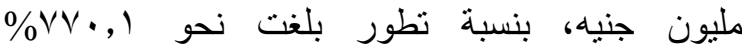

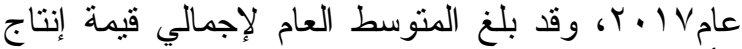

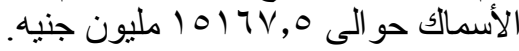

وتتشير تقديرات المعادلة رقم (r) بجدول r ب إلى الإنى معادلة الاتجاه الزمنى العام لنطور قيمة الإنتاج السمكي

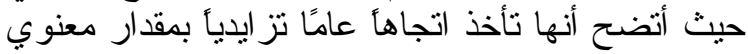

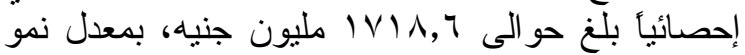
سنوي بلغ نحو ب, 11\%. كما نتشير قيمة معامل التحديد

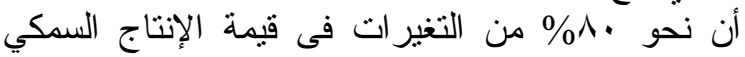

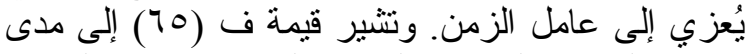

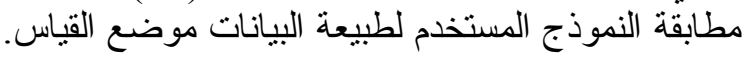

\section{تمهيل}

يعتبر القطاع السمكي أحد القطاعات الإنتاجية الهامة

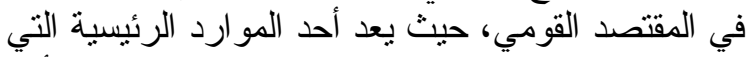

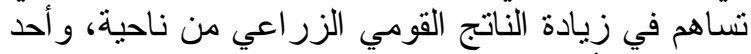

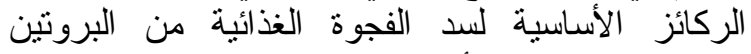

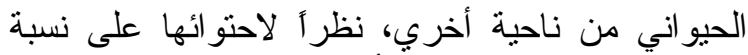

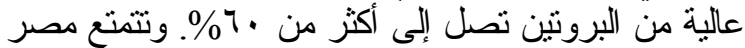

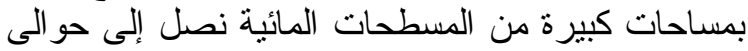
ع ا مليون فدان، بالإضافة إلى العديد من البحير ات اتل مختلفة

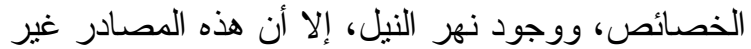

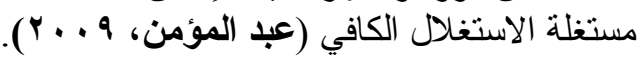
مشكلة البحث البث

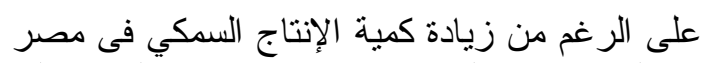

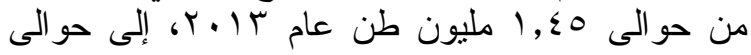

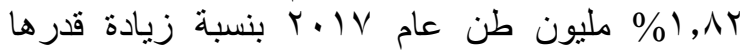
\% ro, .

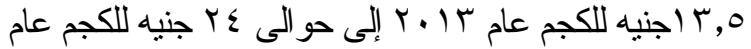

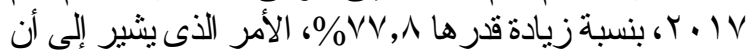

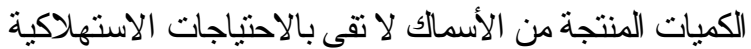

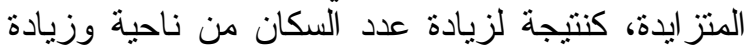

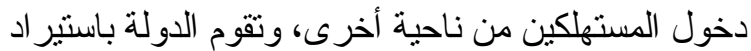

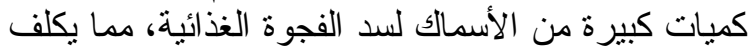

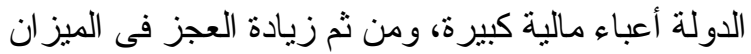

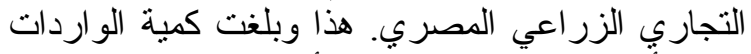

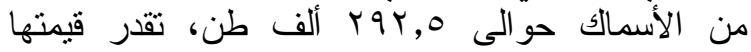

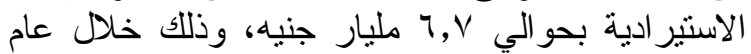
r. 10

\section{هدف البحث}

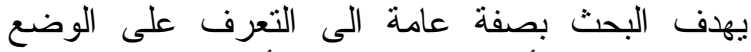
الر اهن لإنتاج الأسماك و المقومات الأساسية التي ينسم بها لأليا

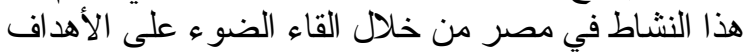

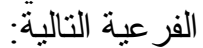

$$
\text { r. ـ در اسة الوضع الوضع الر الهن لإنتاج وقيمة الأسماك في مصر. }
$$

r. استعر اض المقومات الأساسية (مستلزمات الإنتاج) لنشاط إنتاج الأسماك في مصر.

\section{الطريقة البحثية}

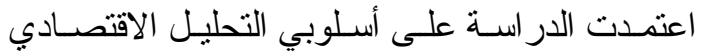

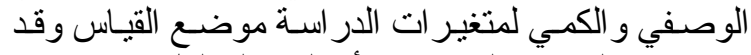

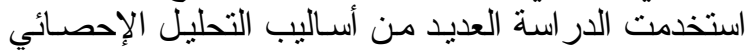

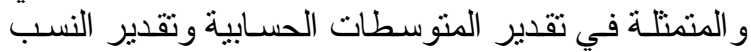

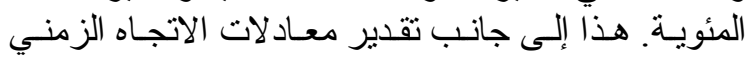


El-Nagar et al. | SINAI Journal of Applied Sciences 9 (1) 2020 105-116

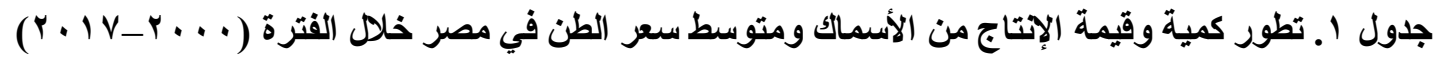

\begin{tabular}{|c|c|c|c|c|c|c|}
\hline $\begin{array}{l}\text { التطور } \\
\text { (\%) }\end{array}$ & م. ملف جنيه) الطن & $\begin{array}{c}\text { التطور } \\
\text { (\%) }\end{array}$ & قليمة الإنتاج) & $\begin{array}{l}\text { التظور } \\
\text { (\%) }\end{array}$ & كمية الإنتاج) & السنوات \\
\hline $1 \ldots$ & $V, \wedge 0$ & $1 \ldots$ & 0717 & $1 \ldots$ & $V Y \varepsilon, r$ & $r \ldots$ \\
\hline $99, \cdot$ & $V, V V$ & $1.0,5$ & 0994,0 & $1.7,0$ & $V \vee 1,7$ & $r \ldots 1$ \\
\hline $9 \wedge, r$ & $\overline{V, V Y}$ & $1 \cdot \Lambda, \wedge$ & $T / \wedge \wedge, r$ & $11 \cdot, v$ & $1 \cdot 1,0$ & $r \ldots r$ \\
\hline $9 V, 7$ & $V, 77$ & $111, \cdot$ & $7 V 1$. & $14 \cdot, 9$ & $\Lambda \vee 7$ & $r \ldots r$ \\
\hline $1 \cdot 9,5$ & 1,09 & $T r \cdot, V$ & $V \leqslant Y \wedge, q$ & 119,8 & 170 & $r \ldots \varepsilon$ \\
\hline IIY,. & $\Lambda, \vee 9$ & $1 \mu V, \varepsilon$ & $V \wedge \mid \varepsilon, 1$ & IYY,A & $\wedge \wedge q, r$ & Y... \\
\hline$\overline{T Y Y, \cdot}$ & 9,01 & $17 \pi, V$ & $94.0, \varepsilon$ & $T r \varepsilon, \cdot$ & $9 V \cdot, 9$ & $r \ldots r$ \\
\hline 117,9 & $1 \cdot, 100$ & $19 \cdot, 0$ & 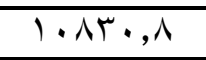 & $1 \Gamma q, r$ & $1 \cdots v, 9$ & $r \ldots V$ \\
\hline $1 \pi 1,7$ & $1 \cdot \pi r$ & 195,0 & $11 \cdot r \cdot, \Lambda$ & $1 \leqslant V, \varepsilon$ & $1.7 V, 7$ & $r \ldots \Lambda$ \\
\hline 1100,9 & $1 \cdot, 7 V$ & $r .0,1$ & $1177 \cdot, 1$ & $10 ., 9$ & $1.94,1$ & $r . . q$ \\
\hline $1 \leqslant 1,0$ & 11,11 & ros, 9 & $1 \leq \leqslant 9 \leqslant, \wedge$ & $1 \wedge \cdot, 1$ & $T \Gamma \cdot \varepsilon, \Lambda$ & $r+1$. \\
\hline $10 V, r$ & IY,To & $r 90,1$ & 17119,1 & $1 \wedge \wedge, 1$ & ITIY, & $r+11$ \\
\hline $17 \pi, \Lambda$ & IY,AT & $\Gamma \cdot, r$ & $1 \vee 7 \varepsilon 1,9$ & $1 \wedge 9, \varepsilon$ & $1 \pi v 1,9$ & $r \cdot I r$ \\
\hline $1 \vee 1, \Lambda$ & $1 r, \leqslant 9$ & $r \leqslant 0, r$ & $197 Y 7,1$ & $r \cdots, \Lambda$ & $1 \leqslant 0 \leqslant, \varepsilon$ & $r \cdot 1 r$ \\
\hline 191,7 & $10, \cdot \varepsilon$ & $r q 1, \lambda$ & YYYA., Z & $r \cdot \varepsilon, T$ & $1 \leq \wedge 1,9$ & $Y .1 \varepsilon$ \\
\hline $197, r$ & 10,51 & $\varepsilon 11, V$ & $r r \varepsilon \cdot \wedge, \wedge$ & $r \cdot q, V$ & 1011,9 & $r+10$ \\
\hline$r \leqslant 1,1$ & $1 \wedge, 94$ & $07 \Lambda, Y$ & $r Y T \cdot V, V$ & YTO,T & $1 V \cdot 7, \varepsilon$ & $r .17$ \\
\hline$r \cdot 7, \cdot$ & $T \varepsilon, \cdot Y$ & $V V \cdot, 1$ & $\varepsilon r \vee \wedge \vee, r$ & $r 01, V$ & IAYY,A & $r+1 V$ \\
\hline - & $11, \wedge r$ & - & $1017 V, 0$ & - & $11 V 1,7$ & المتوسط \\
\hline
\end{tabular}

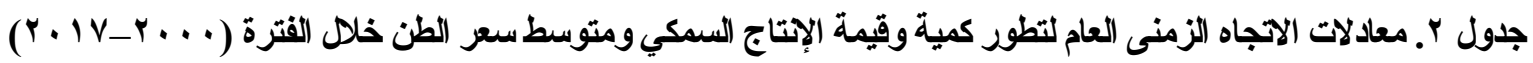

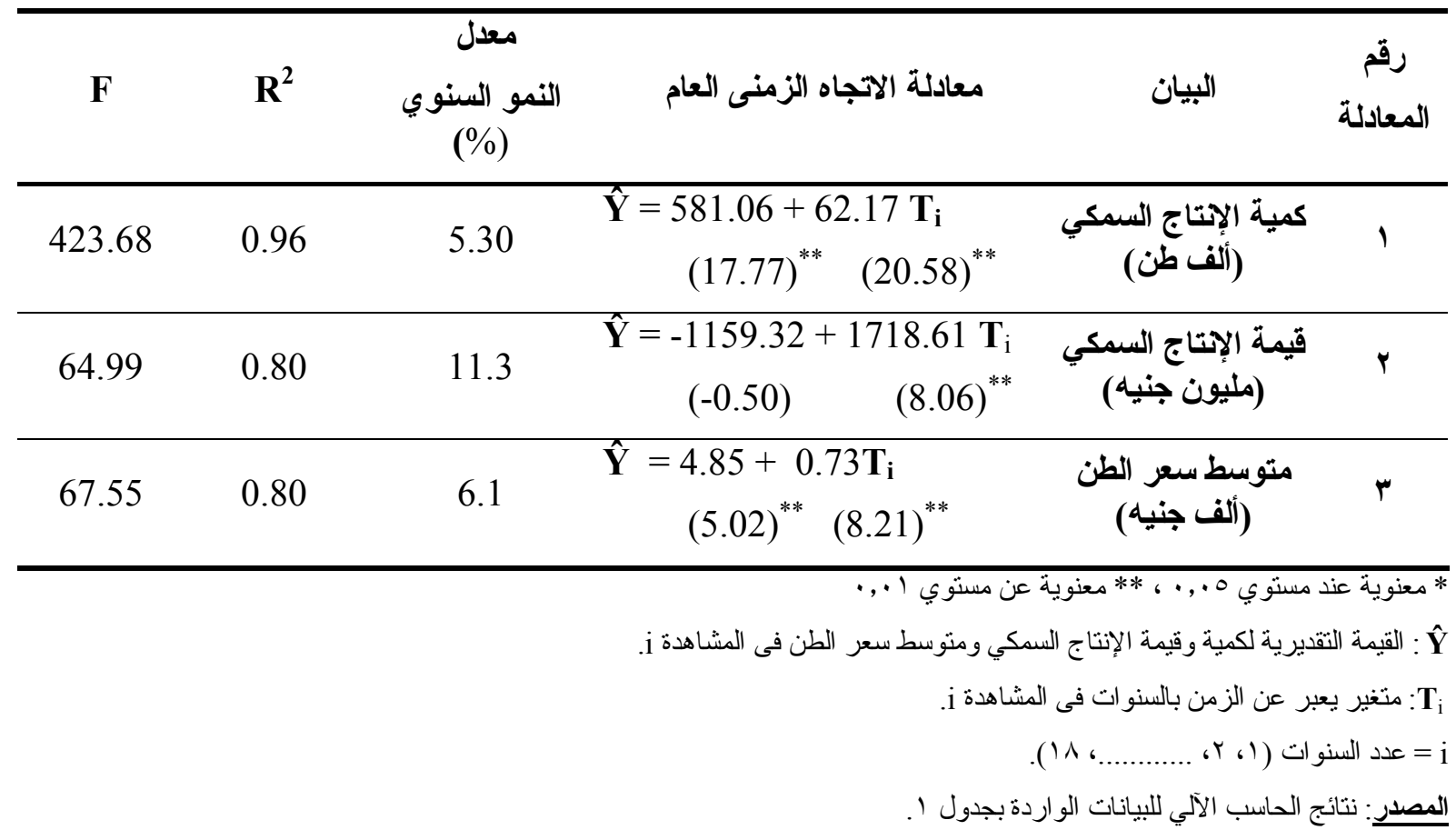




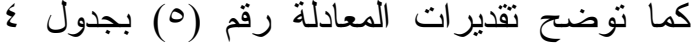

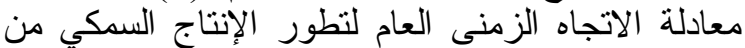

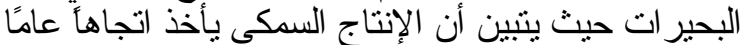

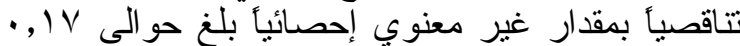

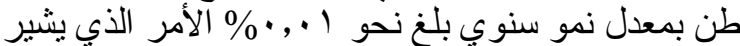

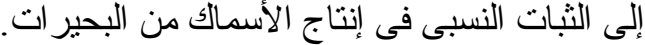

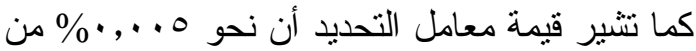

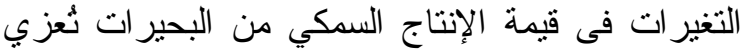

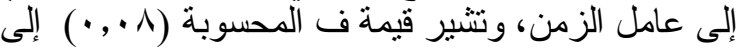
مدى مطابقة النموذج المستخدم لطبيعة البيانات موضع المع

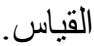

\section{تطور الإتتاج السمكي من المياه العذبة}

أنثارت الأرقام الوارده بجدول ب سابق الآثارة إليه

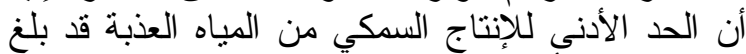

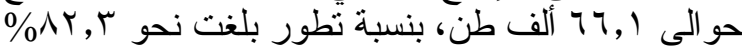

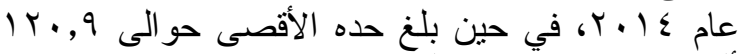

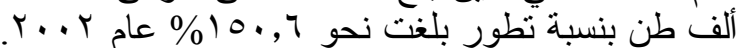
هذا وقد بلغ المنتوط العام للإنتاج السمكي من المن المياه

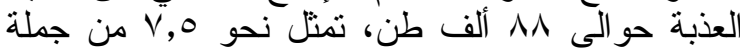

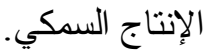

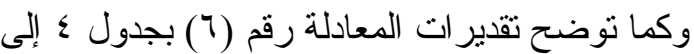

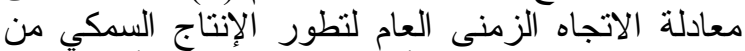

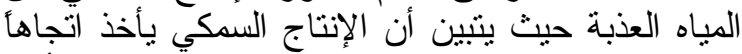

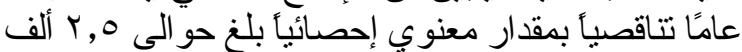

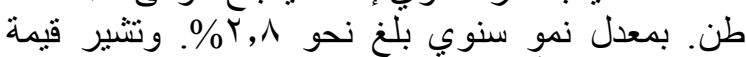

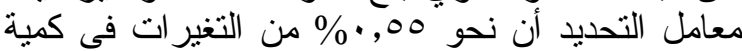

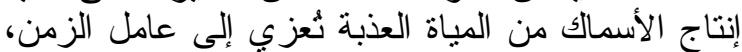

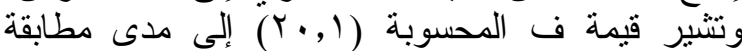

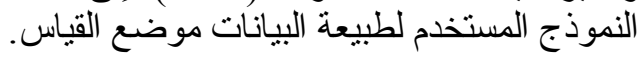

\section{تطور الإتتاج من الاستزراع السمكي}

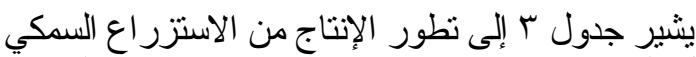

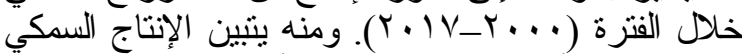

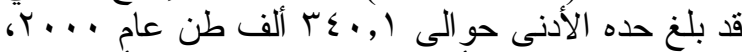

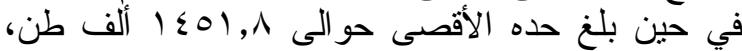
بنسبة تطور بلغت نحو

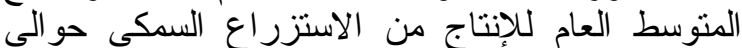

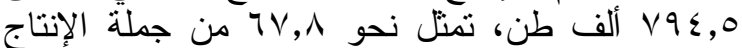

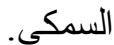

وكما نشير المعادلة رقم (V) بجدول ب إلى معادلة

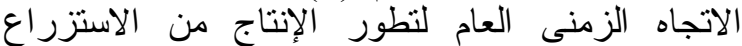

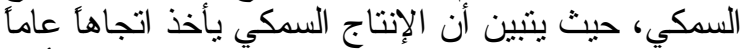

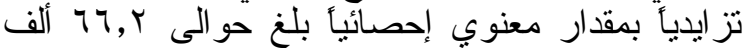

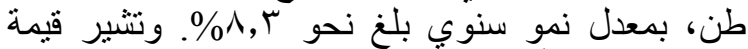

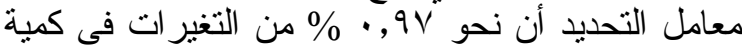

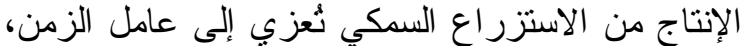

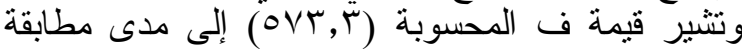
النموذج المستخدم لطبيعة البيانات موضع القياس.
تطور متوسط سعر الطن

باستعر اض الأرقام الواردة بجدول سابق الإثارة

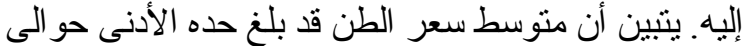

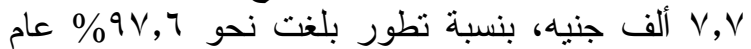

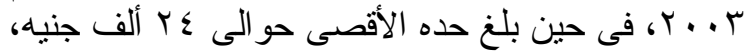

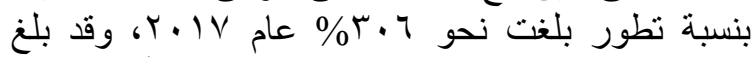

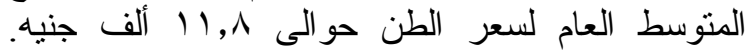

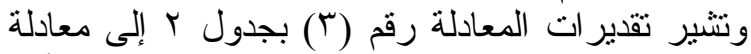

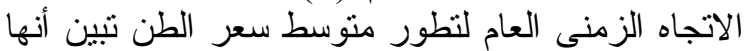

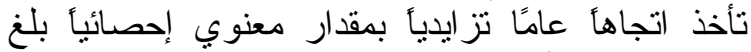

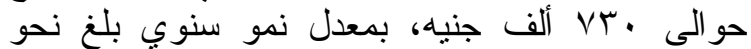

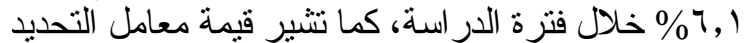

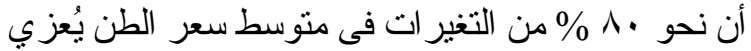

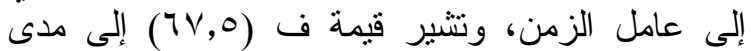
مطابقة النموذج المستخدم لطبيعة البيانات موضع القياس.

\section{تطور إنتاج الأسماك طبقاً للمصايد}

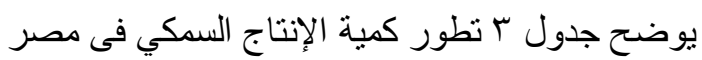

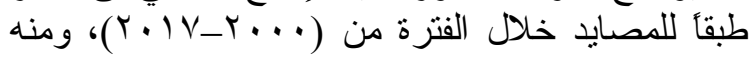

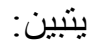

\section{تطور الإنتاج السمكي داخل المياه البحرية}

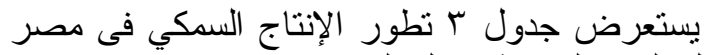

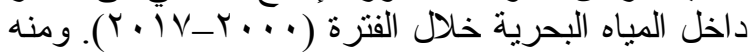

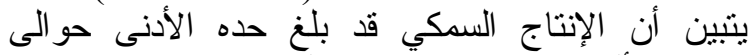

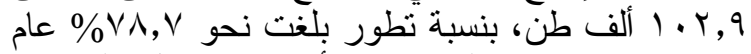

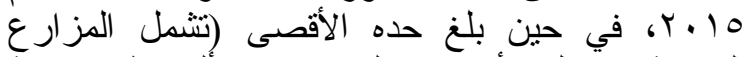

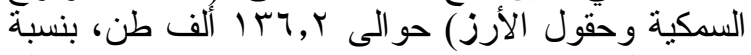

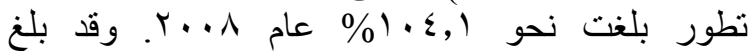

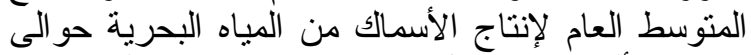

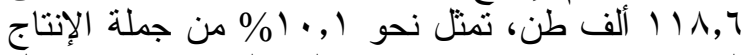

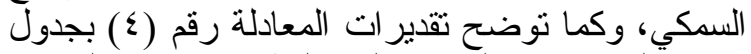

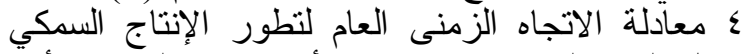

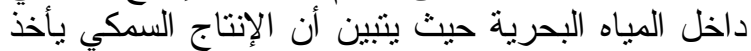

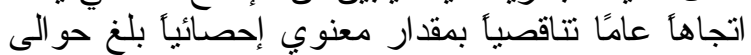

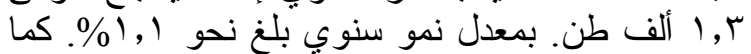

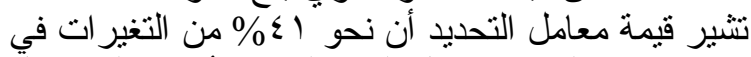

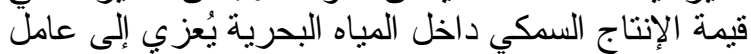

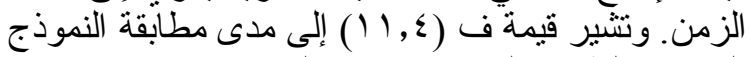

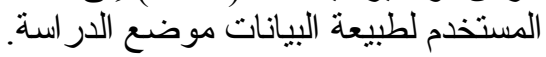

$$
\text { تطور الإنتاج السمكي من البحيرات }
$$

يوضح جدول r تطور الإنتاج السمكي من البحير ات ات التيرن

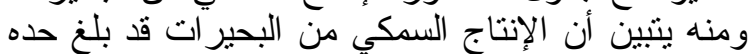

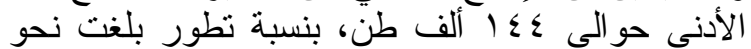

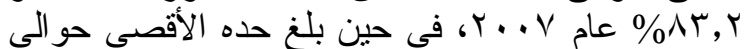

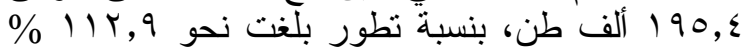

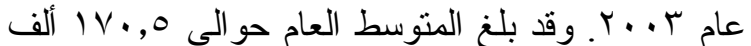
طن، يمثل نحو 7, ؟ـ أ من جملة الإنتاج السمكي. 
El-Nagar et al. | SINAI Journal of Applied Sciences 9 (1) 2020 105-116

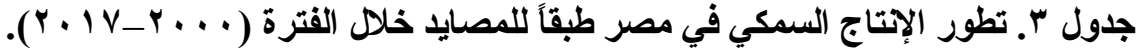

\begin{tabular}{|c|c|c|c|c|c|c|c|c|}
\hline \multicolumn{2}{|c|}{ الاستزراع } & \multicolumn{2}{|c|}{ المياه العذبة } & \multicolumn{2}{|c|}{ البحيرات } & \multicolumn{2}{|c|}{ المياه البحرية } & \multirow[b]{2}{*}{ السنة } \\
\hline $\begin{array}{l}\text { التطور) } \\
\text { (\%) }\end{array}$ & كمية الإنتاج & $\begin{array}{l}\text { التطور } \\
\text { (\%) }\end{array}$ & كمية الإنتاج & $\begin{array}{l}\text { التطور } \\
\text { (\%) }\end{array}$ & كمية الإنتاج & $\begin{array}{l}\text { التطور } \\
\text { (\%) }\end{array}$ & كمية الإنتاج) & \\
\hline $1 \ldots$ & $r \varepsilon \cdot, 1$ & $1 \ldots$ & $\Lambda \cdot, r$ & $1 \ldots$ & IVT,I & $1 \ldots$ & $1 \pi \cdot, \Lambda$ & $r \ldots$ \\
\hline $1 \cdots, \lambda$ & $r \leqslant r, q$ & $1+4,9$ & $1.9,9$ & $1 \cdot V, Y$ & 110,7 & $1 \cdot 1, \lambda$ & ITr,Y & $r \ldots 1$ \\
\hline $11 \cdot, 7$ & rVq, & $10 \cdot, 7$ & $T r \cdot, 9$ & $99, \varepsilon$ & TVY & $1 \cdot 1, r$ & $T r, 0$ & $r \ldots r$ \\
\hline $1 \Gamma \cdot, \lambda$ & $\varepsilon \varepsilon \varepsilon, 9$ & $1 \leqslant \vee, r$ & $11 \wedge, r$ & $11 r, 9$ & $190, \varepsilon$ & $\wedge 9, \wedge$ & $11 V, \varepsilon$ & $r \ldots r$ \\
\hline $1 \Gamma \wedge, \uparrow$ & $\varepsilon \vee 1,0$ & $1 \Gamma \cdot$, & 1.0 & $1 \cdot r, r$ & $1 V V, 1$ & $10, r$ & $111, \varepsilon$ & r.. \\
\hline $10 \Lambda, \mathrm{V}$ & or q,V & $1 \cdot \varepsilon, \varepsilon$ & $\Lambda \mu, \Lambda$ & 91,0 & $10 \Lambda, r$ & $\Delta Y, Y$ & $1 \cdot v, 0$ & $r \ldots o$ \\
\hline $1 V \varepsilon, 9$ & 090 & $1 \Gamma \cdot, \lambda$ & 1.0 & $\lambda \vee, \varepsilon$ & $101, r$ & $91, \varepsilon$ & 119,7 & $r \ldots q$ \\
\hline 117,9 & $7 \pi 0,0$ & $M T, V$ & $9 \vee, V$ & $\Lambda \mu, r$ & $1 \leqslant \varepsilon$ & 99,9 & $T r \cdot, V$ & $r \ldots v$ \\
\hline$r \cdot \varepsilon, \cdot$ & 794,1 & $99, r$ & $\mathrm{Vq}, \mathrm{V}$ & $91, r$ & $10 V, 9$ & $1 \cdot \varepsilon, 1$ & IT4,Y & $r \ldots A$ \\
\hline$r \cdot V, \varepsilon$ & $V \cdot 0,0$ & $1 \cdot \lambda, V$ & $\Lambda V, r$ & 99,0 & IVT,Y & $9 \vee, V$ & $I T V, \Lambda$ & $r \ldots q$ \\
\hline$r V_{\cdot, \varepsilon}$ & 919,7 & $1 \cdot 0, \varepsilon$ & $\Lambda \varepsilon, 7$ & $1 . r, 0$ & $I \vee q, r$ & $9 Y, \Lambda$ & $|Y|, \varepsilon$ & $r \cdot 1$. \\
\hline$r 9 \cdot, 1$ & $9 \wedge 7, \wedge$ & $111, \mathrm{~V}$ & $\Lambda 9, \vee$ & $9 \leqslant, \Gamma$ & 17r,r & 94.0 & TYY,r & $r \cdot 11$ \\
\hline rq9,Y & $1.1 \mathrm{~V}, \mathrm{~V}$ & $\Delta r, q$ & 77,7 & $1 \cdots, r$ & $I V T, \varepsilon$ & $\Lambda V, r$ & $11 \varepsilon, r$ & $r \cdot I r$ \\
\hline rYY,Y & $1.9 V, 0$ & $\Lambda \varepsilon, \Gamma$ & $T V, V$ & $1.0, \varepsilon$ & IAY,O & 11,7 & $1 \cdot 7, v$ & $r \cdot I r$ \\
\hline$r \Psi \varepsilon, r$ & $11 r v, 1$ & $\Delta r, r$ & 77,1 & $9 \wedge, \vee$ & $1 V_{\cdot, 9}$ & $\Delta r, \varepsilon$ & $1 \cdot \vee, \Lambda$ & $r \cdot 1 \leqslant$ \\
\hline$r \leqslant 0, \varepsilon$ & $11 V \varepsilon, \wedge$ & $\Lambda 7, \wedge$ & 79,1 & 99,1 & $|V|, 0$ & $\vee \wedge, \vee$ & $1 \cdot Y, 9$ & $r .10$ \\
\hline$\varepsilon \cdot \Gamma, \cdot$ & $T r V \cdot, V$ & 91,0 & $V \Psi, 0$ & 91,7 & 101,0 & $V q, r$ & I.r,V & $r .17$ \\
\hline \multirow[t]{3}{*}{$\sum$ \&४, } & $1 \leqslant 01,1$ & 97,1 & $V V, V$ & $1.7,4$ & $1 \wedge \Gamma, 0$ & $\Delta r, q$ & $1.9,1$ & $Y \cdot I V$ \\
\hline & $V 9 \leqslant, 0$ & & $\wedge \vee, q$ & & $I V \cdot, 0$ & & $11 \wedge, 7$ & المتوسط \\
\hline & $T V, A Y$ & & $v, 01$ & & $1 \varepsilon, 00$ & & $1 \cdot, I r$ & $(\%)$ \\
\hline
\end{tabular}

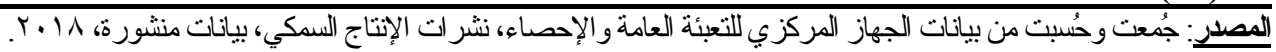

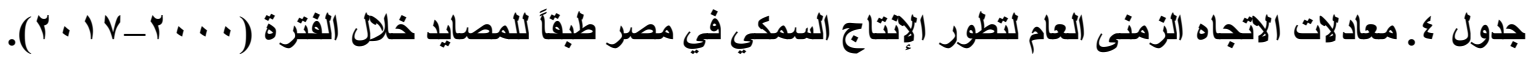

\begin{tabular}{|c|c|c|c|c|c|}
\hline $\mathbf{F}$ & $\mathbf{R}^{2}$ & 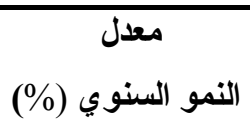 & معادلة الاتجاه الزمنى العام & أنواع المصايد & رقادلة \\
\hline 11.43 & 0.41 & (1.13) & $\begin{array}{l}\hat{\mathbf{Y}}=131.48-1.35 \mathbf{T}_{\mathbf{i}} \\
(30.42)^{* *}(-3.38)^{* *}\end{array}$ & المياة البحرية & $\varepsilon$ \\
\hline 0.08 & 0.005 & $(0.01)$ & $\begin{array}{c}\hat{Y}=172.22-0.17 \mathbf{T}_{\mathbf{i}} \\
(26.19)^{* *}(-0.29)^{* *}\end{array}$ & البحيرات & 0 \\
\hline 20.09 & 0.55 & $(2.80)$ & $\begin{array}{c}\hat{\mathbf{Y}}=111.44-2.47 \mathbf{T}_{\mathbf{i}} \\
\quad(18.67)^{* *}(-4.48)^{* *}\end{array}$ & المياة العذبة & 7 \\
\hline 573.26 & 0.97 & 8.32 & $\begin{aligned} \hat{\mathbf{Y}}= & 165.91+66.16 \mathbf{T}_{\mathbf{i}} \\
& (5.54)^{* *} \quad(23.94)^{* *}\end{aligned}$ & الاستزراع السمكي & $v$ \\
\hline
\end{tabular}




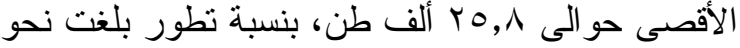

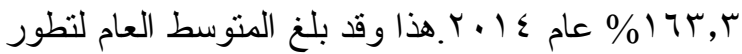

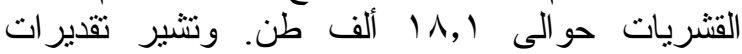

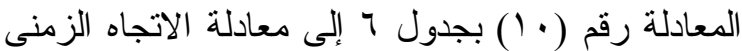

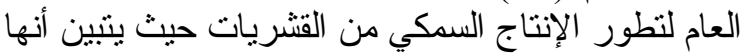

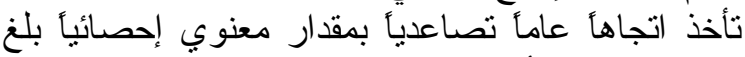

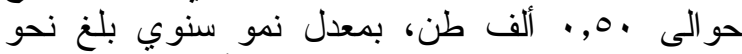

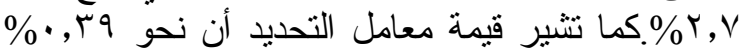

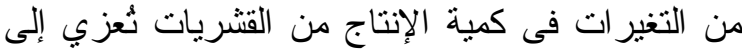

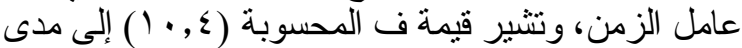

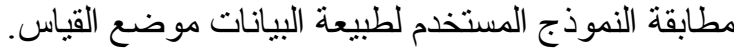

\section{تطور الرخويات}

تثير الأرقام الواردة بجدول ه نطور الإتتاج السمكي

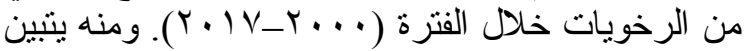

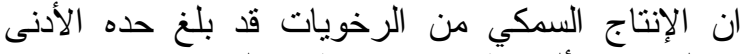

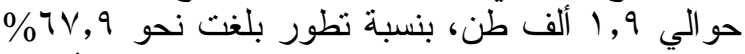

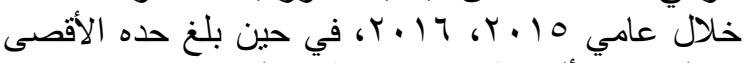

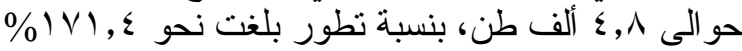

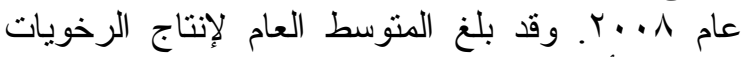

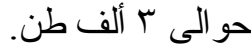

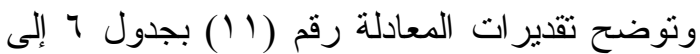
معادلة الاتجاه الزمنى العام لتطور الإنتاج السمكي من لته

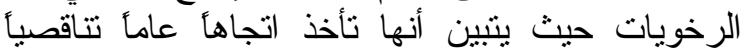

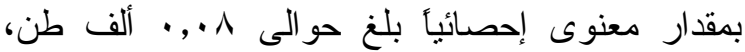

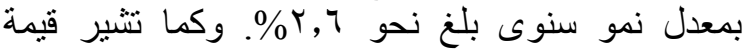

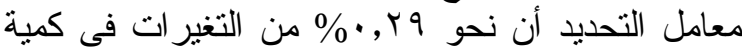

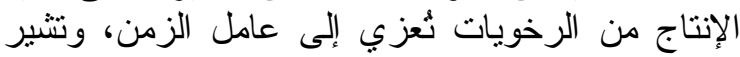

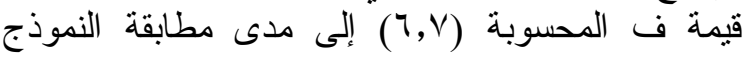
المستخدم لطبيعة البيانات موضع القياس.

\section{تطور الرئويات}

يوضح جدول ه نطور الإنتاج السمكي من الرئويات

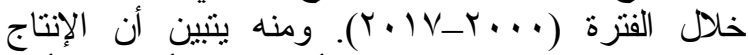

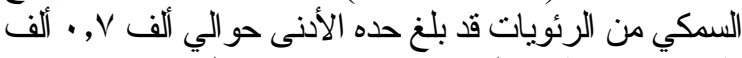

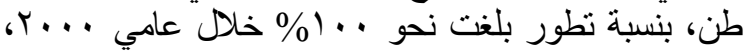

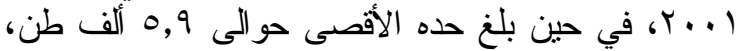

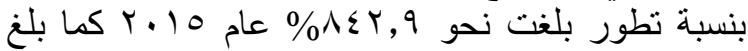

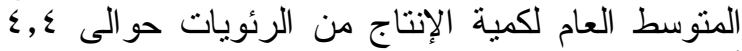

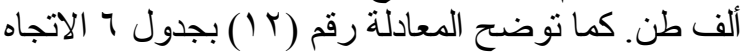

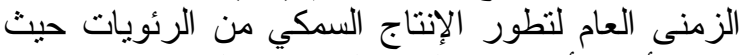

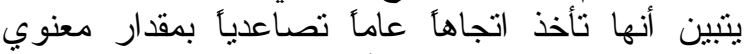

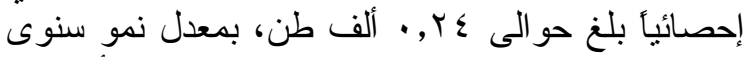

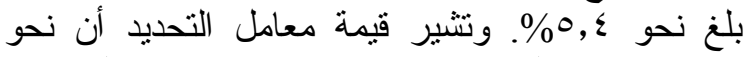

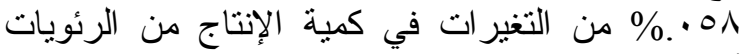

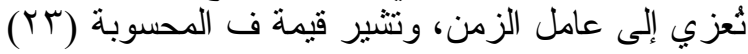
إلى مدى مطابقة النموذج المستخدم لطبيعة البيانات

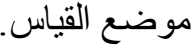

ثالثاً: تطور الإتتاج السمكي طبقاً للمجموعات السمكية:

يوضح جدول ه تطور الإنتاج السمكي طبقأ

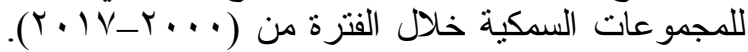

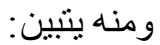

\section{تطور الأسماك العظمية}

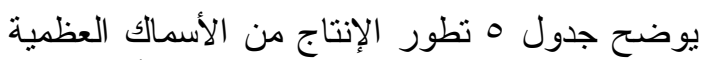

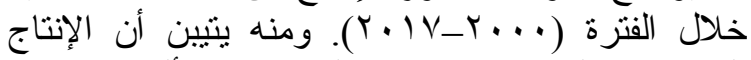

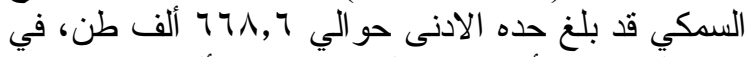

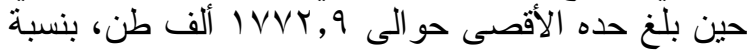

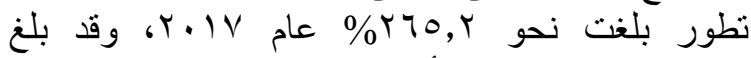

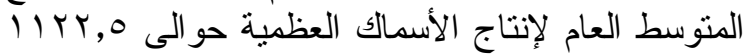

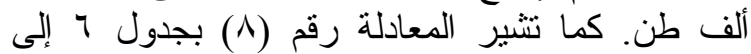
معادلة الاتجاه الزمنى العام لنطور الإنتاج السمكي من الإندي

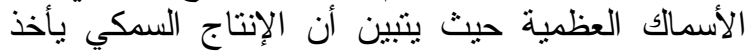

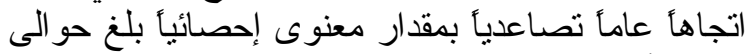

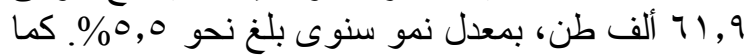

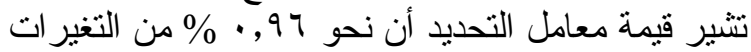

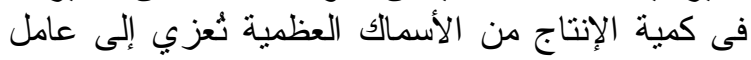

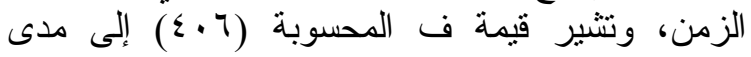

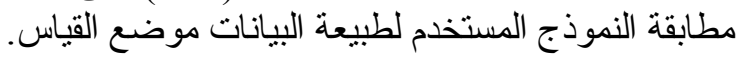

\section{تطور الأسماك الغضروفية}

تشير الأرقام الواردة بجدول ه إلى تطور الإنى الإنتاج

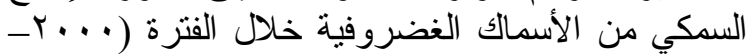

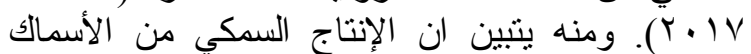

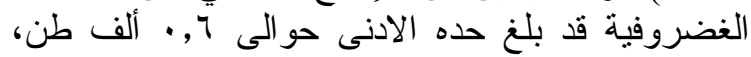

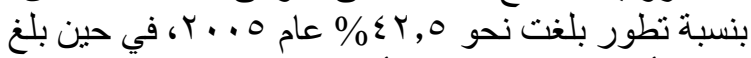

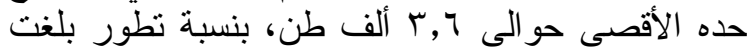

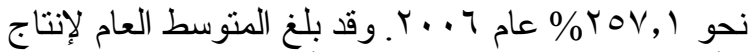

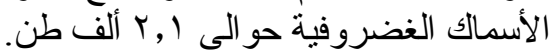

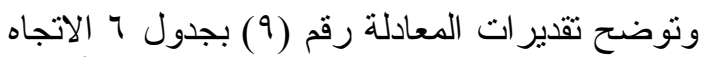

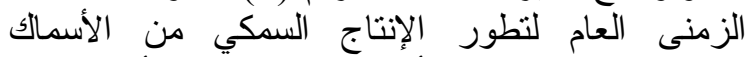

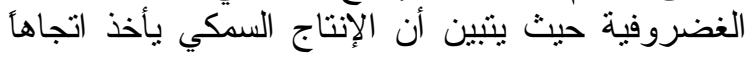

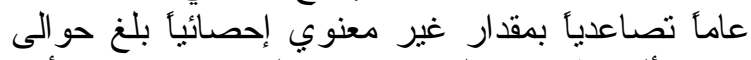

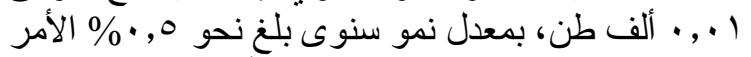
الذي يشير إلى الثبات النسبى لإنتاج الأسماك الغضرو نهروفية.

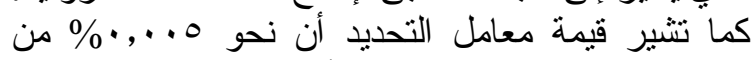

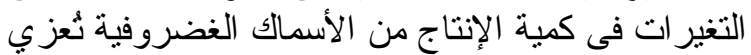

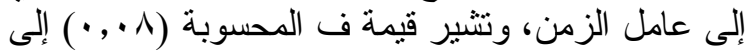
مدى مطابقة النموذج المستخدم لطبيعة البيانات موضع فئح

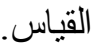

\section{تظور القشريات}

بالإثارة إلي الأرقام الواردة بجدول ه سابق الإثـارة

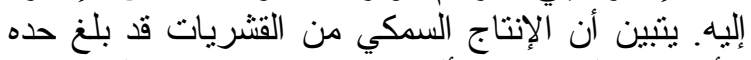

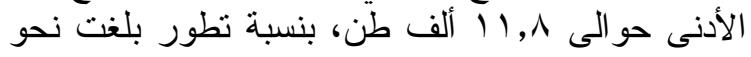
\% \&,V 


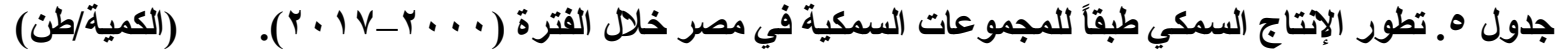

\begin{tabular}{|c|c|c|c|c|c|c|c|c|c|c|c|c|c|}
\hline \multirow{2}{*}{ (ألف طالن) } & \multicolumn{2}{|c|}{ أخرى } & \multicolumn{2}{|c|}{ الرئويات } & \multicolumn{2}{|c|}{ الرخويات } & \multicolumn{2}{|c|}{ القثريات } & \multicolumn{2}{|c|}{ الأسماك الغضروفية } & \multicolumn{2}{|c|}{ الأسماكك العظمية } & \multirow[b]{2}{*}{ السنة } \\
\hline & $\begin{array}{l}\text { التطور } \\
\text { (\%) }\end{array}$ & كمبة الإنتاج & $\begin{array}{l}\text { التطور } \\
\text { (\%) }\end{array}$ & كمبة الإنتاج) & $\begin{array}{l}\text { التطور } \\
\text { (\%) }\end{array}$ & 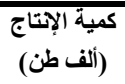 & $\begin{array}{l}\text { التطور) } \\
\text { (\%) }\end{array}$ & كمية الإنتاج) & $\begin{array}{l}\text { التطور } \\
\text { (\%) }\end{array}$ & كمية الإنتاج & $\begin{array}{l}\text { التطور } \\
\text { (\%) }\end{array}$ & كمية الإتتاج & \\
\hline$V Y \leq, r$ & $1 \ldots$ & ro & $1 \ldots$ & $\cdot, \mathrm{V}$ & $1 \ldots$ & $\overline{r, \Lambda}$ & $1 \ldots$ & 10,1 & $1 \ldots$ & $1, \xi$ & $1 \ldots$ & $74 \Lambda, 7$ & r... \\
\hline VVI, T & $v \wedge, r$ & $r V, \varepsilon$ & $1 \ldots$ & ., $\mathrm{v}$ & $11, \mathrm{v}$ & $r, 1$ & $V \leqslant, v$ & $11, \wedge$ & $|V|,\{$ & $r, \varepsilon$ & $1 \cdot \Lambda, 7$ & VYY,Y & $r \ldots l$ \\
\hline$\Lambda \cdot 1,0$ & $\checkmark \Lambda, q$ & $r \leqslant, 1$ & $1 \% \Lambda, 4$ &., 9 & $1 \ldots$, & $r, \Lambda$ & $\Lambda \leqslant, \wedge$ & | $1 \%$ & $10 \mathrm{~V}, 1$ & $r, r$ & & $v \otimes \wedge, 1$ & $r \ldots r$ \\
\hline AV & 00,1 & $19, r$ & vov, 1 & $0, r$ & $10 .,$. & $\varepsilon, r$ & $v \leqslant, v$ & 11,1 & $11 \leqslant, r$ & 1,7 & $I r \leqslant, V$ & גזr, & $r \ldots r$ \\
\hline م & $\varepsilon$ & $1 \leqslant, 1$ & IVI, & $\varepsilon, v$ & $11 \mathrm{v}, 9$ & $r, r$ & $\wedge \wedge,$. & 17,9 & $1 \ldots$, & $1, \varepsilon$ & Irr, & $\Lambda Y V, r$ & r... \\
\hline$\wedge \wedge q, r$ & תו, & $r \pi, 1$ & $\Delta \wedge \theta, V$ & $\{, 1$ & $1 . v, 1$ & $r$ & $11 \cdot, \wedge$ & IV,o & $\leqslant r, q$ & ז, & $1 Y 0, \Lambda$ & $\Lambda \leqslant 1$ & r...o \\
\hline$q \vee \cdot, q$ & (1), & 11,7 & VYA, T & 0,1 & $|r|, \varepsilon$ & r, & १४,० & $10, \varepsilon$ & rov, 1 & ૫, & $\mid r \Lambda, r$ & $Q Y \leq, \Lambda$ & $r \ldots r$ \\
\hline $1 \cdots v, q$ & 0., r & $1 v, 7$ & rov, & ד, § & $10 ., 0$ & $\varepsilon, Y$ & $1 \cdot v, r$ & IV & IVA, 7 & $r, 0$ & $1 \leqslant r, q$ & & $r \ldots v$ \\
\hline $1.78,7$ & 11,1 & $r u, \xi$ & $v \ldots$, & \&, , & $|v|, \varepsilon$ & $\{, \wedge$ & $1 \leqslant \wedge, 1$ & $r \mu, \varepsilon$ & rY, & $r, 1$ & 101,1 & 1.1. & $r \ldots \wedge$ \\
\hline $1.94,1$ & $10, v$ & rT & $v ו \varepsilon, r$ & 。 & I & $r, r$ & $10 \%, 4$ & $r \leqslant, q$ & iv^, & r,o & $10 \leqslant, V$ & I. & r...q \\
\hline 1 & $r, .$, & r) & $v ו \leqslant, r$ & 。 & $11 v, q$ & $r, r$ & A & $r, r$ & rY, \& & $r, 1$ & $|A v|$, & 1 ro1,1 & $r .1$. \\
\hline ITry, & $V Y, q$ & $r_{0,0}$ & $v 1 \leqslant, r$ & - & $1 . r, 4$ & r, q & $1+0, \varepsilon$ & $r 1, \varepsilon$ & $r M O, V$ & $r, r$ & $190,$. & $1 T \cdot \varepsilon$ & r.11 \\
\hline$|r v|, q$ & , & YY, & $\checkmark \wedge \theta, v$ & , ^ & १४, & $r, v$ & $1.1,9$ & 17,1 & $17 \leqslant, r$ & $r, r$ & $19 \wedge,$. & דry & $r .1 Y$ \\
\hline $1 \leqslant 0 \leqslant, \leqslant$ & or,q & 11,0 & $\wedge \ldots, \cdot$ & 0,4 & Ar,l & $r, r$ & \& & r $1, \varepsilon$ & $10 .,$. & $r, 1$ & $r 1 ., 1$ & 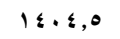 & $r .1 r$ \\
\hline $1 \leqslant \wedge 1, q$ & $00, v$ & 19,0 & $v \wedge \diamond, v$ & 0,0 & $\wedge q, r$ & $r, 0$ & r, & ro, 1 & $\mid r \wedge, \tau$ & 1,1 & \& & $1 \leqslant r \varangle, 1$ & $r+1 \leqslant$ \\
\hline 1011,9 & 0,1 & $i v, v$ & $\Lambda \leqslant r, q$ & 0,9 & iv, q & 1,9 & $11 \mathrm{v}, \mathrm{v}$ & $1 \wedge, 7$ & $\vee \wedge, \tau$ & 1,1 & YY, E & $1 \leqslant V r, V$ & $r .10$ \\
\hline $1 V \cdot 4, \varepsilon$ & $\varepsilon v, v$ & $17, V$ & $\wedge \ldots, \cdot$ & 0,7 & $7 v, q$ & 1,9 & $1 \cdot \Lambda, r$ & $1 v, 1$ & QY, q & $1, r$ & $r \leqslant \Lambda, \Lambda$ & 1784,1 & Y.17 \\
\hline IArr,A & ד & 19,1 & $\Lambda$ A & $\bullet, \wedge$ & $v_{0},$. & $r, 1$ & $1+1,4$ & $r \cdot, \wedge$ & $1 \ldots$, & $1, \varepsilon$ & rTo,r & IVVY,q & $r .1 \mathrm{~V}$ \\
\hline $11 \times 1,0$ & & $r, r$ & & $\{, \varepsilon$ & & $r, \cdot r$ & & $\mid \Lambda, 1$ & & $r, . q$ & & 11rr,t & لمتوسط \\
\hline $1 \ldots$ & & $1, A r$ & & . , זA & & דצ', & & 1,00 & &., 11 & & $90, \wedge$ & (\%) \\
\hline
\end{tabular}

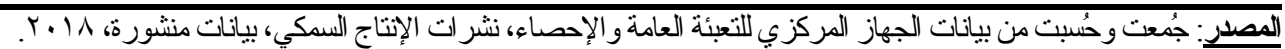

\section{تطور عدد مر اكب الصيا

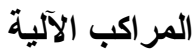

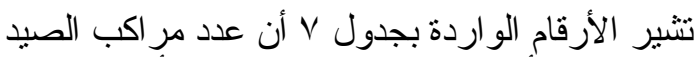

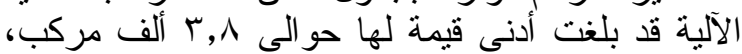

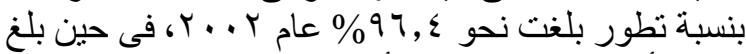

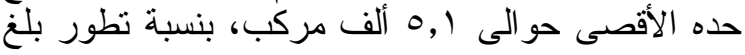

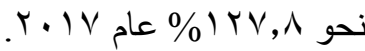

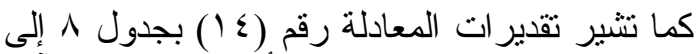

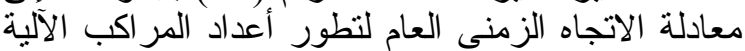

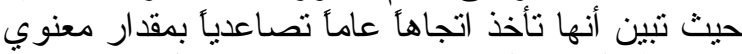

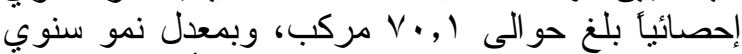

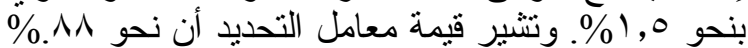

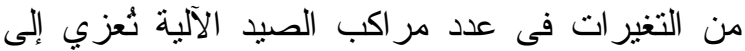

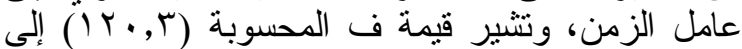
مدى مطابقة النموذج المستخدم لطبيعة البيانات موضع بلى القياس.

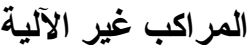

يتضح من جدول V أن عدد مر اكب الصيد غير الآلية

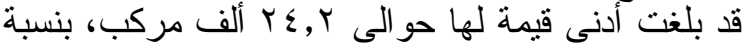

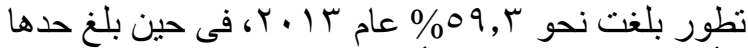

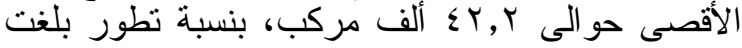

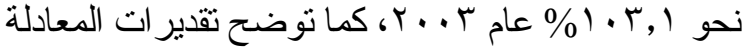

الأصناف الأخرى

بالإثارة إلي الأرقام الواردة بجدول ه سابق الإثـارة

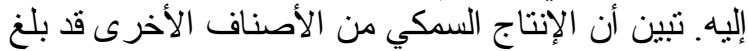

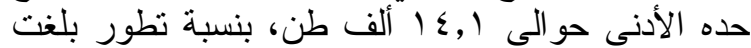

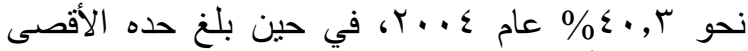

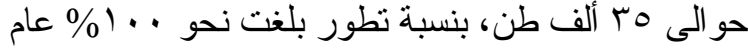

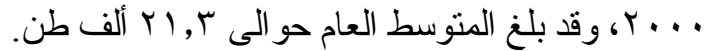

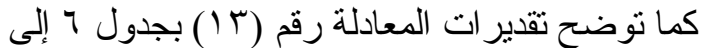

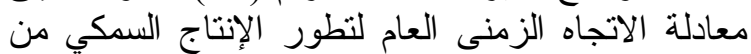

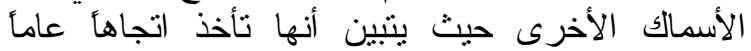

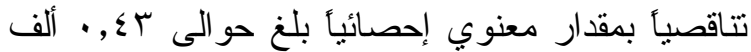

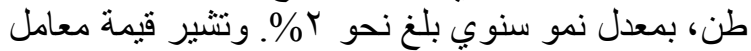

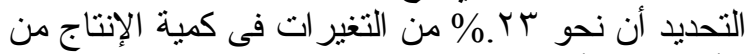

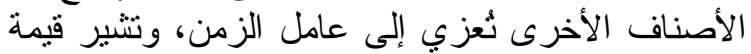
ف المحسوبة (0) إلى مدى مطابقة النموذج المئي المستخدم لطبيعة البيانات موضع القياس.

المقومات الأساسية (مستلزمات الإتتاج) داخل نشـاط فئاط إنتاج الأسماك في مصر الأسنة

تتير الأرقام الواردة بجدول V للمقومات الأساسية

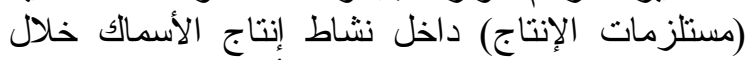

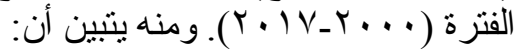


El-Nagar et al. | SINAI Journal of Applied Sciences 9 (1) 2020 105-116

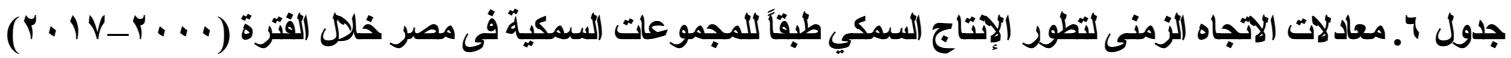

\begin{tabular}{|c|c|c|c|c|c|}
\hline $\mathbf{F}$ & $\mathbf{R}^{2}$ & $\begin{array}{c}\text { النمو السنوي } \\
\text { (\%) }\end{array}$ & معادلة الاتجاه الزمنى العام & المجموعات السمكية & المعادلة \\
\hline 405.95 & 0.96 & 5.5 & $\begin{aligned} \hat{\mathbf{Y}}= & 534,15+61.94 \mathbf{T}_{\mathbf{i}} \\
& (16.05)^{* *}(20.14)^{* *}\end{aligned}$ & الأسماك العظمية & $\wedge$ \\
\hline 0.08 & 0.005 & $(0.5)$ & $\begin{array}{c}\hat{\mathbf{Y}}=2.19-0.011 \mathbf{T}_{\mathbf{i}} \\
(5.21)^{* *}(-0.28)^{* *}\end{array}$ & الأسماك الغضروفية & 9 \\
\hline 10.44 & 0.39 & 2.7 & 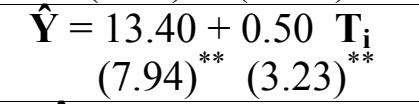 & القشريات & 1. \\
\hline 6.73 & 0.29 & (2.6) & $\begin{array}{c}\hat{\mathbf{Y}}=3.78-0.08 \mathbf{T}_{\mathbf{i}} \\
\quad(11.25)^{* *}(-2.59)^{* *}\end{array}$ & 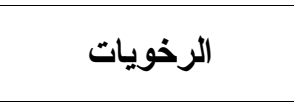 & 11 \\
\hline 22.95 & 0.58 & 5.4 & $\begin{array}{c}\hat{\mathbf{Y}}=2.03+0.24 \mathbf{T}_{\mathbf{i}} \\
(3.61)^{* *}(4.79)^{* *}\end{array}$ & الرئويات & Ir \\
\hline 4.97 & 0.23 & $(2.01)$ & $\begin{array}{r}\hat{\mathbf{Y}}=25.47-0.43 \mathbf{T}_{\mathrm{i}} \\
(12.14)^{* *}(-.2 .23)^{* *}\end{array}$ & أخرى & $1 r$ \\
\hline
\end{tabular}

جدول V. أعداد مر اكب الصيد و أعداد الصيادين و أعداد الجمعيات، وقيمة الوقود و الزيوت داخل نشاط إنتاج الأسماك في

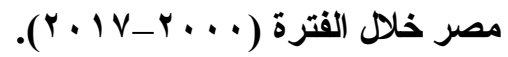

\begin{tabular}{|c|c|c|c|c|c|c|c|c|c|c|c|c|c|c|c|c|}
\hline \multicolumn{2}{|c|}{ الوقود و الزيوت } & \multicolumn{6}{|c|}{ الجمعيات } & \multicolumn{2}{|c|}{ المرخصيادين } & \multicolumn{6}{|c|}{ عدد مراكب الصبا } & \multirow{3}{*}{ السنوات } \\
\hline \multirow{2}{*}{ التطور } & \multirow{2}{*}{ (الفيمة } & \multicolumn{2}{|c|}{ الإجمالي } & \multicolumn{2}{|c|}{ استزراع } & \multicolumn{2}{|c|}{ محلية } & \multirow{2}{*}{ التطور } & \multirow[b]{2}{*}{ العدد العد } & \multirow{2}{*}{$\begin{array}{l}\text { التطور } \\
\text { (\%) }\end{array}$} & \multirow{2}{*}{ المر اكبة } & \multicolumn{2}{|c|}{ غير آلية } & \multicolumn{2}{|c|}{ آلية } & \\
\hline & & $\begin{array}{l}\text { التطور } \\
\text { (\%) }\end{array}$ & العدد & $\begin{array}{l}\text { التطور } \\
\text { (\%) }\end{array}$ & العدد & $\begin{array}{l}\text { التطور } \\
\text { (\%) }\end{array}$ & العدد العد & & & & & $\begin{array}{l}\text { (\%) } \\
\text { (\%) }\end{array}$ & العدد & $\begin{array}{l}\text { التطور } \\
\text { (\%) }\end{array}$ & العدد & \\
\hline $1 \cdots$ & $9 r v q$ & $1 \ldots$ & $\Lambda V$ & $1 \cdots$ & V & $1 \ldots$ & $\Lambda$. & $1 \cdots$ & 9.490 & $99,9$. & «ะ१ा. & $1 \ldots$ & $\{.909$ & $1 \ldots$ & $r q 0 \leqslant$ & $r \ldots$ \\
\hline $0, \Lambda$ & $1178 v$ & $1 \ldots$ & $\Lambda \vee$ & $1 \ldots$ & $v$ & $1 \ldots$ & $\Lambda$. & $q \uparrow, \varepsilon$ & OOAKV & $9 \wedge, \xi$. & $\varepsilon \leqslant \wedge \uparrow \varepsilon$ & $99, q$ & $\{.91$. & $1 \ldots$ & rq० & $\ldots 1$ \\
\hline r. & $1 \leqslant \Lambda V$. & $1 \ldots$ & $\Lambda \mathrm{V}$ & $1 \ldots$ & $v$ & $1 \ldots$ & $\wedge$. & $v \varepsilon, v$ & $\leqslant 0.9$. & $1 \cdot r, 11$ & $\leq \leqslant 191$ & $9 \wedge, \uparrow$ & $\varepsilon \cdot r \vee q$ & $9 \%, \xi$ & rAIr & $r \ldots r$ \\
\hline$\cdot r, r$ & $9 \leq q \leq$ & $1,1,1 \varepsilon$ & $\wedge \wedge$ & $1 \ldots$ & v & $1 \cdot 1, r$ & $\Lambda_{1}$ & $V \Psi, \varepsilon$ & $\leqslant \varepsilon r . q$ & $\wedge \wedge, 1 \leq$ & \& & $1, r, 1$ & \&YYIA & $1 . r, \varepsilon$ & $\varepsilon \cdot \wedge q$ & $r \ldots r$ \\
\hline $1 \wedge r, q$ & IV.TY & I. & 9. & $1 Y \wedge, 7$ & 9 & $1 \cdot 1, r$ & 1) & IV, I & $\varepsilon . \Delta \Delta r$ & $\vee \wedge, \vee \uparrow$ & rq०Ar & ヘษ,r & rorr. & $1 \cdot v, 0$ & $\varepsilon r \otimes r$ & $r \ldots \varepsilon$ \\
\hline 178,7 & 10000 & I.r, $\{\leq$ & 9 . & $1 Y \wedge, \tau$ & $q$ & $1 \cdot 1, r$ & $\Lambda_{1}$ & $r \wedge, q$ & $\{1719$ & $74, .1$ & rorv. & $v \bullet, v$ & $r . q \wedge \vee$ & $11 \cdot, 1$ & $\varepsilon r \wedge r$ & $r \ldots o$ \\
\hline $1 \wedge \wedge, 0$ & $|v \leqslant q|$ & I. r, $\{\leq$ & 9. & $1 Y \wedge, 7$ & 9 & $1 \cdot 1, r$ & 1) & $v \cdot, v$ & 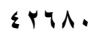 & $7 \varepsilon, 79$ & Yq & צ & $r 0100$ & $11 \%, 7$ & $\leq \leqslant q$. & $r \ldots r$ \\
\hline $1 \wedge \wedge, 0$ & $|v \leqslant q|$ & $1 . \leqslant, 09$ & 91 & $1 \leqslant r, q$ & 1. & $1 \cdot 1, r$ & Al & $v \vee, q$ & $\leqslant V .0 \leqslant$ & $T V, \Lambda$. & rq.or & $\Lambda \varepsilon, r$ & $r \leqslant 01$. & $11 \leq, 9$ & $\leqslant 0 \leqslant r$ & $r \ldots v$ \\
\hline 174,7 & 10 & $1.7, \wedge 9$ & 94 & $10 v, 1$ & 11 & $1, r, 0$ & Ar & $\wedge r, 1$ & $0 . r 11$ & $\vee \vee, \wedge q$ & $r . \leqslant \leqslant q$ & Y Y, & Y०Y . & $|r|, 7$ & $\leqslant 1.9$ & $r \ldots \Lambda$ \\
\hline 9,1 & $r V \leqslant V I$ & $\| \mu, V$ & 99 & $10 \mathrm{~V}, 1$ & 11 & $11, .$. & $\Lambda \wedge$ & $r_{0,1}$ & $0 V \leqslant 04$ & $\vee \wedge, 1$. & ए १q ४q & $V r, q$ & $r . r v i$ & 119,1 & $\varepsilon v \cdot \Lambda$ & $r \ldots q$ \\
\hline$\leqslant I V, \Lambda$ & rAvVI & $11 \leq, 9$ & $1 \ldots$ & 108,1 & 11 & $111, r$ & $\wedge q$ & $\Delta, r, r$ & PII & $10, \vee \wedge$ & $r 0 . V \varepsilon$ & $V r, q$ & $r . r \leqslant \Lambda$ & Irr, & \&AY & $r \cdot 1$. \\
\hline$v \bullet \bullet, 7$ & $v \cdot 11$. & $114, v 9$ & 99 & $1 \leqslant r, q$ & 1. & $111, r$ & $\wedge 9$ & OV,O & $r \leqslant V r V$ & $\neg \wedge, \curlyvee \leq$ & $r q 0 \leqslant r$ & $7 ., r$ & $r \leq 7 q 1$ & Irr,V & $\varepsilon \wedge \circ r$ & $r \cdot 11$ \\
\hline V५ & 811.7 & $111, \leqslant 9$ & १४ & $1 \leqslant r, q$ & 1. & $1 \cdot \Lambda, \Lambda$ & $\wedge \vee$ & ז & משת & $\checkmark \varepsilon, \wedge q$ & r.ArA & rT, & r०q१q & $\mid r \varepsilon, r$ & $\varepsilon 9.9$ & $r \cdot I r$ \\
\hline $97 \varepsilon, 9$ & NqOrr & $111, \leqslant 9$ & १४ & $1 \leqslant r, q$ & 1. & $1 \cdot \Lambda, \Lambda$ & $\wedge \vee$ & $r v, l$ & rrmq & Y Y, V0 & $r q \mid \leq \varepsilon$ & $\bullet q, r$ & $r \leqslant r \wedge$. & Irr,. & $\leq \wedge 7 \leqslant$ & $r .1 r$ \\
\hline $97 \varepsilon, 9$ & NQOrr & |Y,T & $9 \wedge$ & $10 \mathrm{~V}, 1$ & 11 & $1 \cdot \Lambda, \Lambda$ & $\Lambda V$ & $\{1,0$ & ro. $\Lambda$. & $\uparrow \wedge, \uparrow \wedge$ & 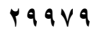 & $71, \varepsilon$ & $r 010$. & Irr, & \&Arq & $r \cdot 1 \leq$ \\
\hline$\wedge \uparrow 0, \uparrow$ & A. 19 & $11 r, v q$ & 99 & $1 \leqslant r, q$ & 1. & $111, r$ & $\wedge 9$ & $\Delta q, 0$ & roq. & $r 0, r r$ & $r \cdot \Lambda \leqslant r$ & ז,rי & roqrT & $\mid r \leq \varepsilon$ & $\leqslant 919$ & $r .10$ \\
\hline Vฯ & 211.7 & $117, .9$ & 1.1 & $|V|, \varepsilon$ & ir & $111, r$ & $\wedge 9$ & $01,$. & $r \cdot v \wedge \Delta$ & VY,qT & צqur & 09,0 & $r \leqslant r \wedge 1$ & $1, r o, r$ & $\varepsilon 900$ & $r .18$ \\
\hline Аाт,. & vovir & $117, .9$ & 1.1 & $1 \wedge \theta, v$ & ir & $11 .,$. & $\wedge \wedge$ & $7 \wedge, 9$ & \&ITrY & $\vee \wedge, \vee \wedge$ & rrvos & $7 \vee, 7$ & rvV. 1 & $I r v, \Lambda$ & 0.04 & $r+I V$ \\
\hline
\end{tabular}


El-Nagar et al. | SINAI Journal of Applied Sciences 9 (1) 2020 105-116

جدول ^. معادلات الاتجاه الزمنى للمقومات الأساسية (مستلزمات الإتتاج) داخل نثاط إنتاج الأسماك فى مصر خلال

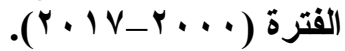

\begin{tabular}{|c|c|c|c|c|c|}
\hline $\mathbf{F}$ & $\mathbf{R}^{2}$ & 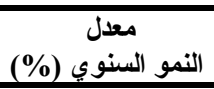 & معادلة الآجاه الزمنى العام & مقومات الإتاج السمكي & رقمعادلة \\
\hline 120.35 & 0.88 & 1.5 & 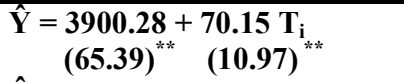 & عدد المر اكب الآلية (مركب) & $1 \leqslant$ \\
\hline 36.95 & 0.69 & (3.3) & 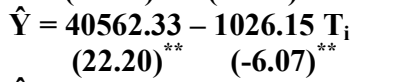 & المر اكب غير الآلية (مركب) & 10 \\
\hline 33.66 & 0.67 & (2.7) & $\begin{aligned} & \hat{Y}= 44462.61-956.01 T_{i} \\
&(24.92)^{* k+} \\
&(-5.80)^{*+*}\end{aligned}$ & جملة مراكب الصيد (مركب) & 18 \\
\hline 13.97 & 0.46 & (3.4) & $\hat{\mathbf{Y}}=\begin{aligned} 5423.37-1418.26 T_{i} \\
(13.20)^{* * *} \\
(-3.73)^{* * *}\end{aligned}$ & عدد الصيادين المرخصين & iv \\
\hline 58.66 & 0.78 & 0.7 & $\begin{aligned} \hat{\mathbf{Y}}= & 78.36+0.64 \mathrm{~T}_{\mathrm{i}} \\
& (86.66)^{* * *}(7.65)^{* * *}\end{aligned}$ & عدد الجمعيات المحلية & 11 \\
\hline 53.78 & 0.77 & (2.9) & $\hat{\mathbf{Y}}=\begin{aligned} 5423.37-1418.26 T_{i} \\
(13.20)^{* * *}\end{aligned}$ & عدد جمعيات الاستزراع السمكي & 19 \\
\hline 108.64 & 0.87 & 0.9 & $\begin{aligned} \hat{Y}= & 85.24+0.93 T_{i} \\
& (87.99)^{* * *}(10.42)^{* * *}\end{aligned}$ & جمله الجمعيات & r. \\
\hline 67.15 & 0.80 & 12.9 & $\begin{aligned} \hat{\mathbf{Y}}= & =\mathbf{- 9 0 9 0 . 1 3}+5296.28 \mathrm{~T}_{\mathrm{i}} \\
(-1.29)^{* * *} & (8.19)^{* * *}\end{aligned}$ & 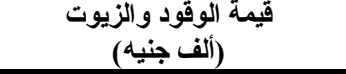 & $r_{1}$ \\
\hline
\end{tabular}

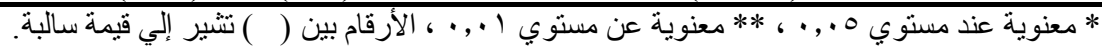
T

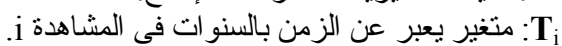

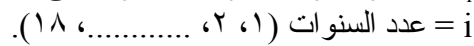

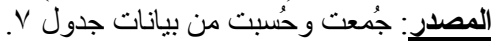

\section{عداد الصيادين المرخصين}

بالاشارة إلي الأرقام الو اردة بجدول V سابق الإنشارة

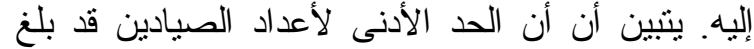

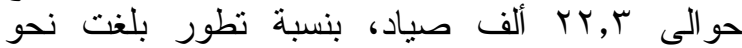
\&rV,

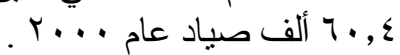

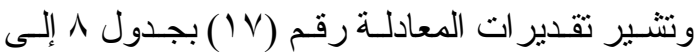

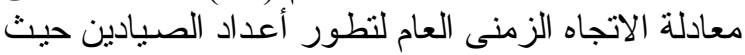

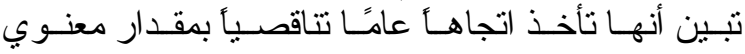

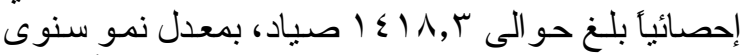

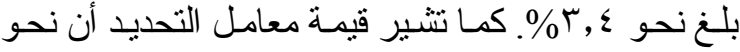

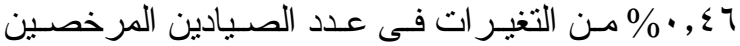

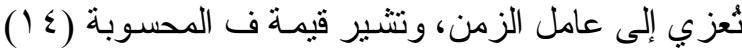

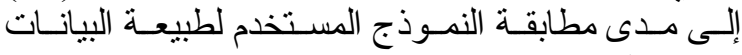

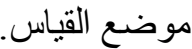

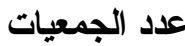

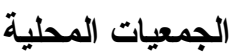

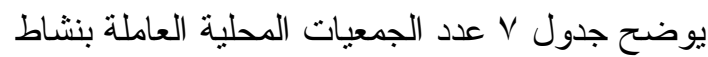

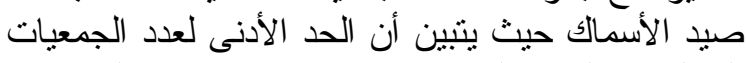

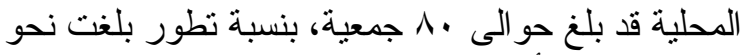

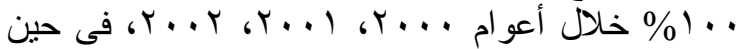

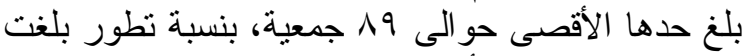

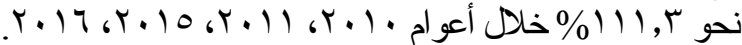

رقم (10) بجدول ^ معادلة الاتجاه الزمنى العام لتطور

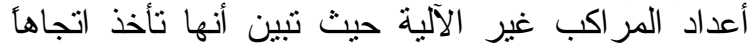

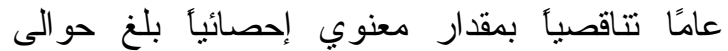

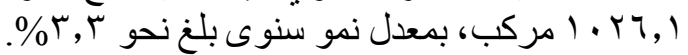

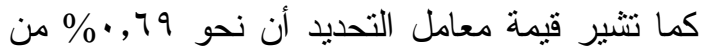

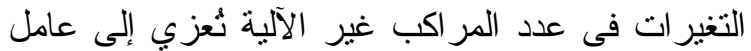

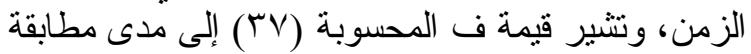
النموذج المستخدم لطبيعة البيانات موضع القياس.

$$
\text { جملة مر اكب الصيا }
$$

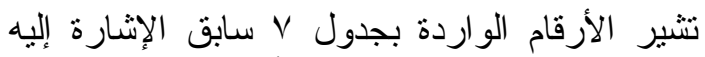

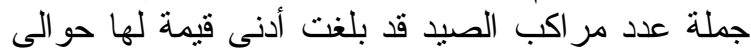

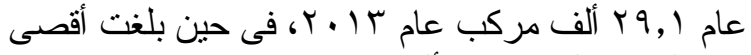

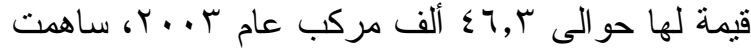

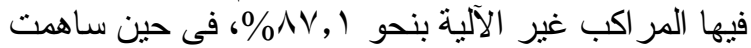

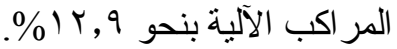

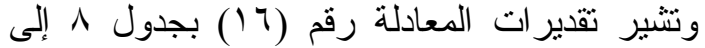

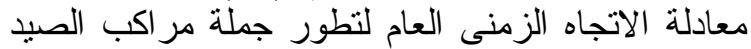

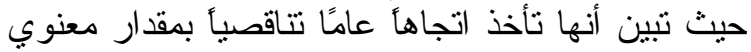

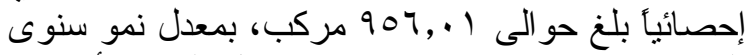

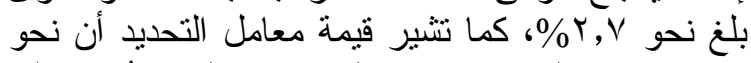

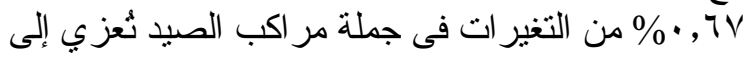

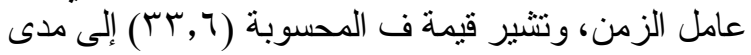
مطابقة النموذج المستخدم لطبيعة البيانات موضع القياس. 


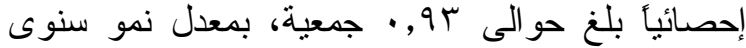

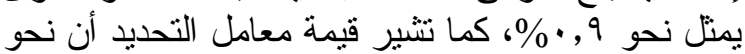
ع •, AV

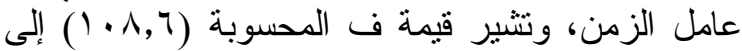

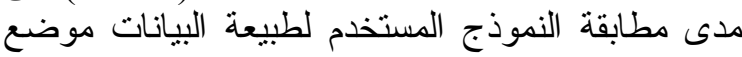
القياس.

\section{الوقود و الزيوت}

تشير الأرقام الو اردة بجدول V أن الحد الأدنى للوقود

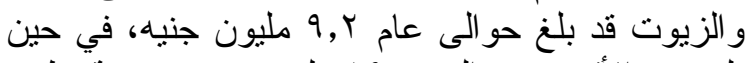

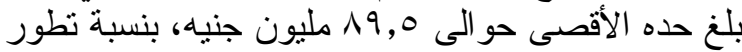

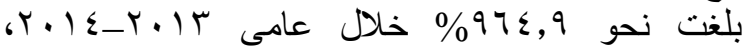

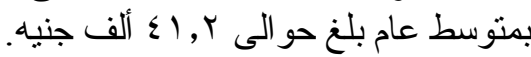

وتوضح تقدير ات المعادلة رقم (Yl) بجدول 1 در اسة

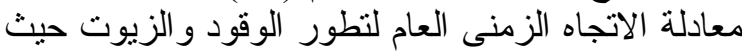

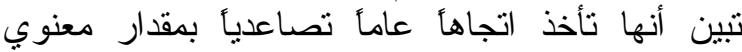

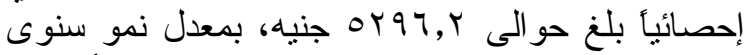

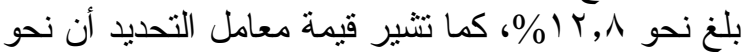

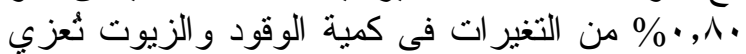

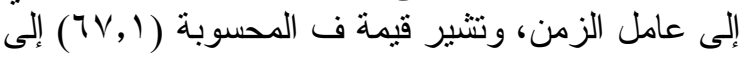

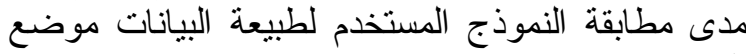

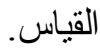$$
\text { المراجـاج }
$$

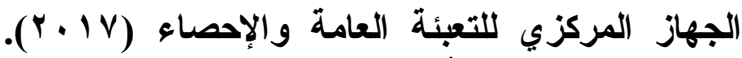 كتاب مصر فى أرقام.

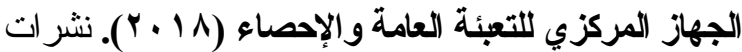 الإنتاج السمكي، بيانات منشورة المرئه}

رضوان، رياض إسماعيل مصطفى (Y I • Y). المنظور

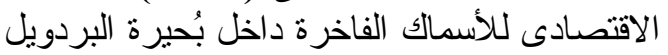

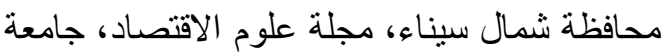
المنصورة، مجلد (ب)، عدد (1) (1).

عبدالمؤمن، شعبان عبدالجيد (9 . . Y). در اسة اقتصادية

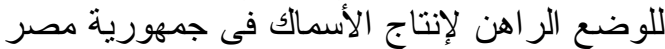

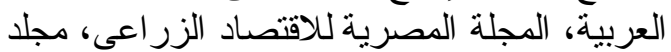

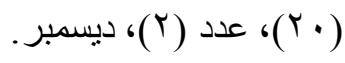

وتوضح تقدير ات المعادلة رقم (1 ا ) بجدول 1 معادلة الاتجاه الزمنى العام لتطور أعداد الجمعيات المحلية حيث

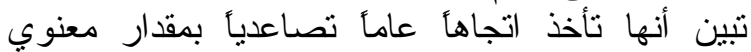

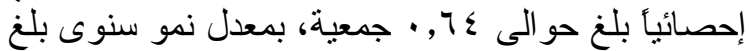

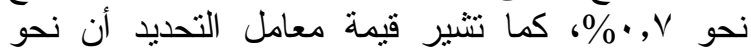

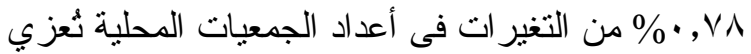

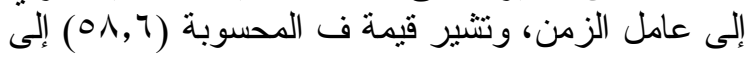
مدى مطابقة النموذج المسنتخدم لطبيعة البيانات موضع فئع

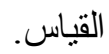

\section{جمعيات الاستزر اع السمكي}

بالاشارة إلي الأرقام الواردة بجدول V سابق الإشارة

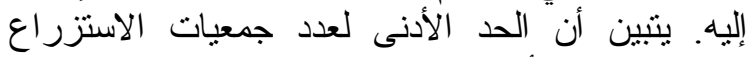

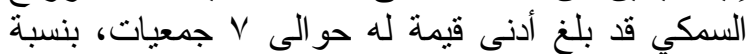

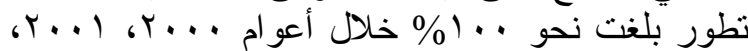

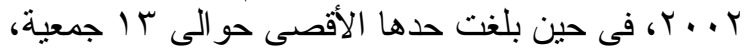

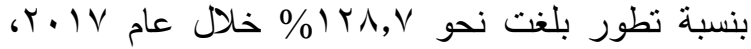

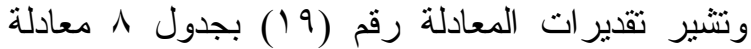

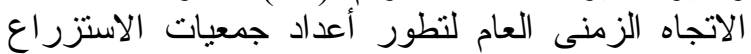

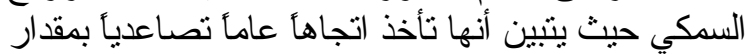

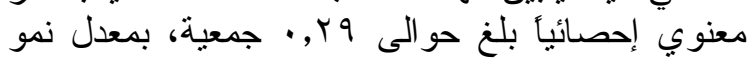

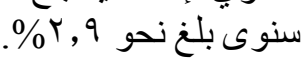

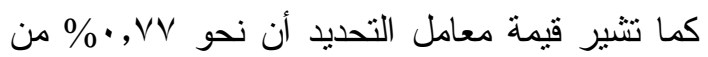

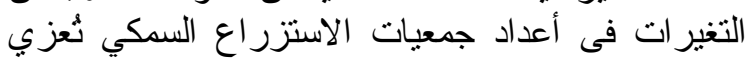

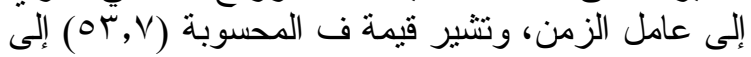

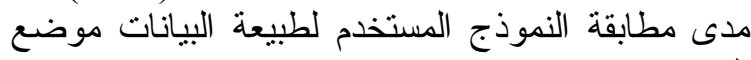

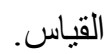

\section{جملة عدد الجمعيات}

يتبين من جدول V أن الحد الأدنى لجمله عدد الجمعيات

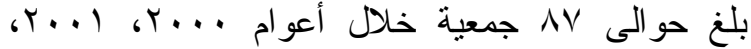

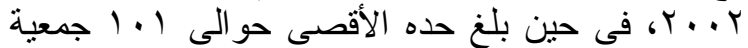

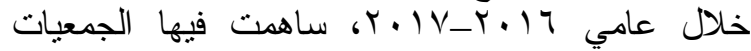

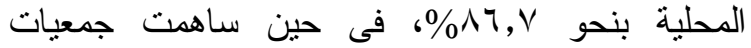

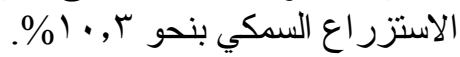

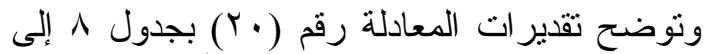

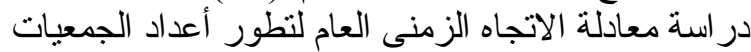

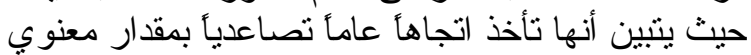




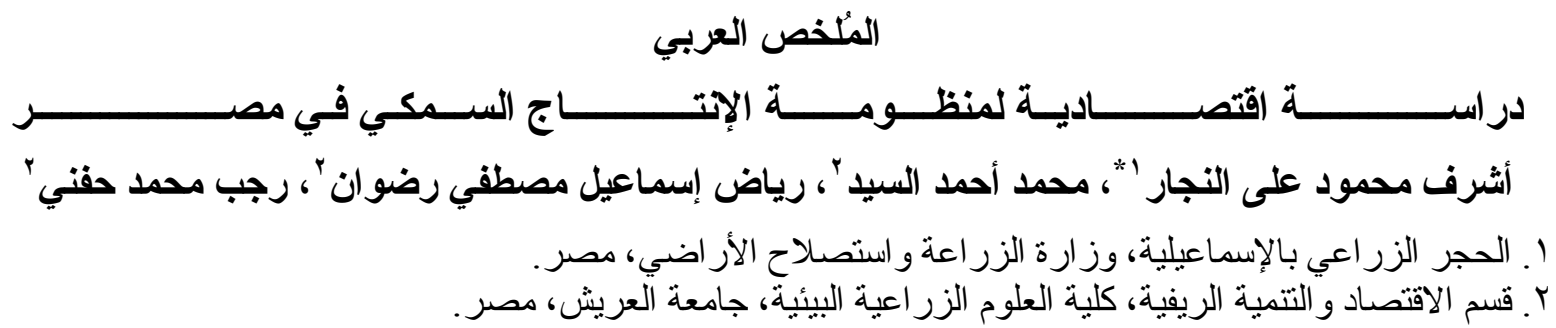

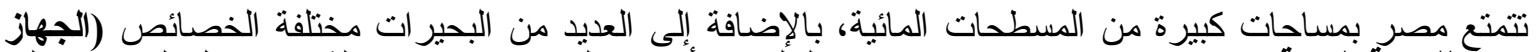

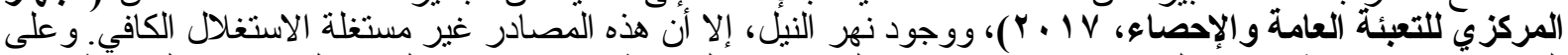

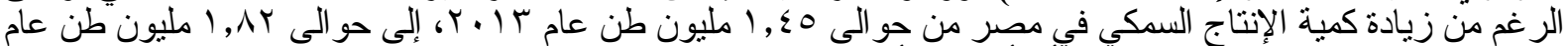

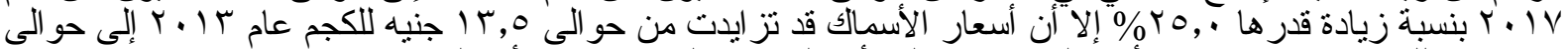

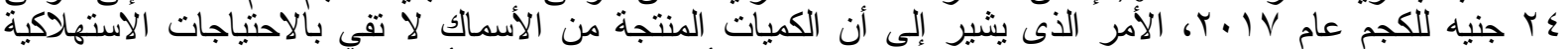

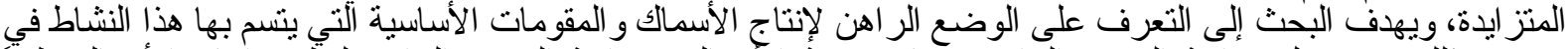

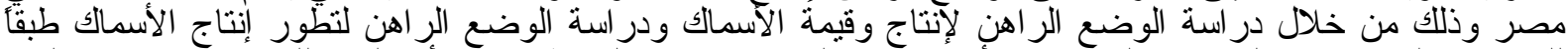

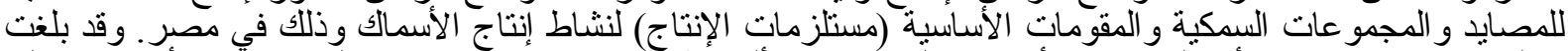

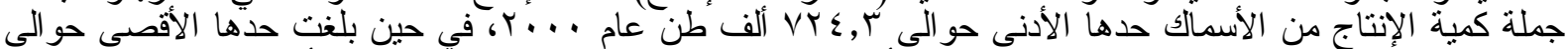

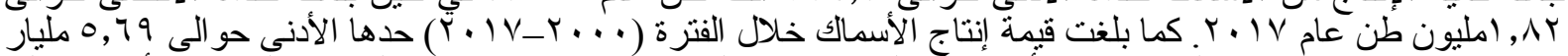

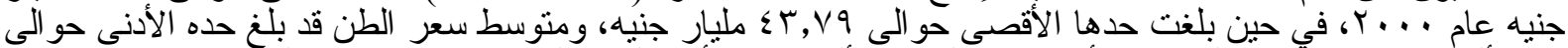
V V,

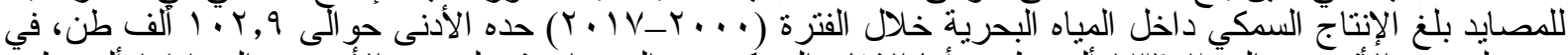

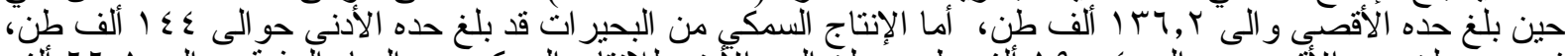

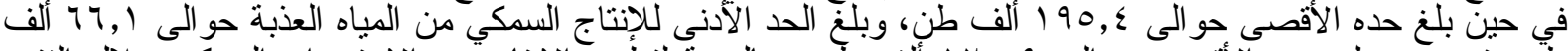

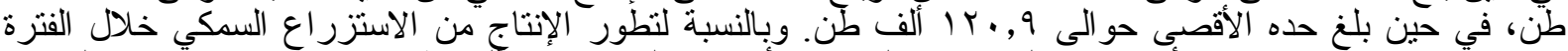

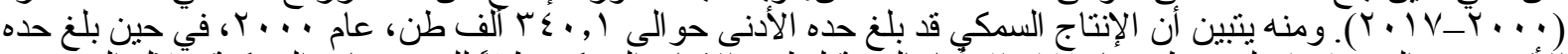

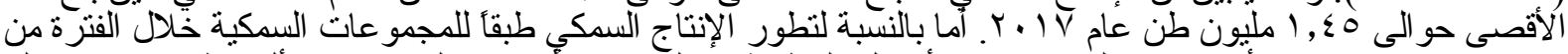

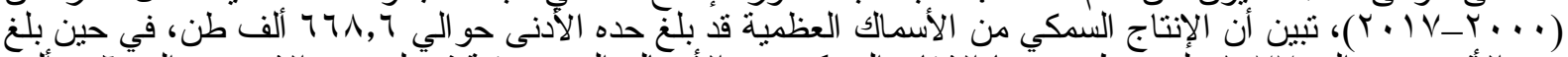

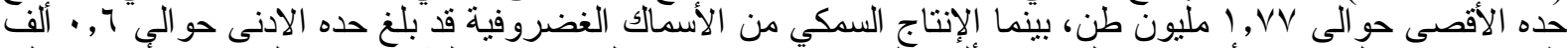

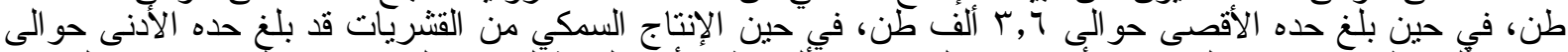

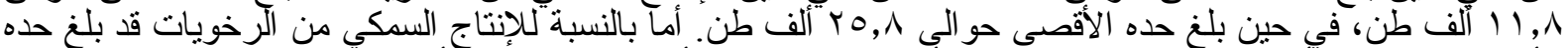

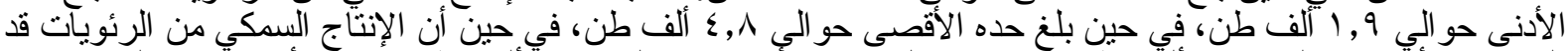

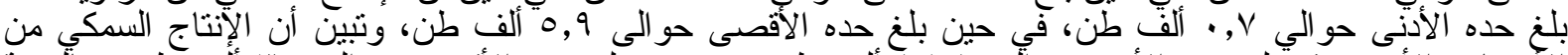

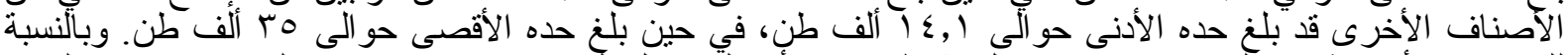

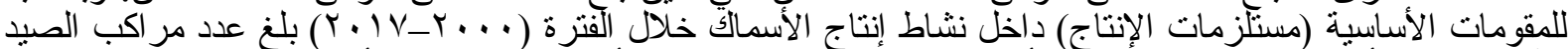

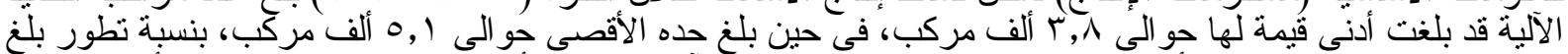

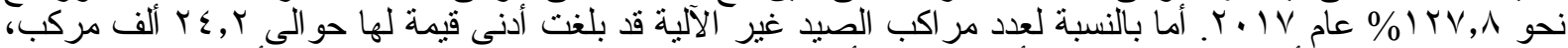

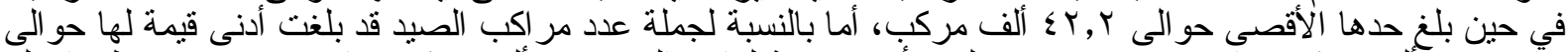

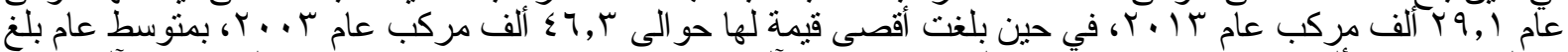

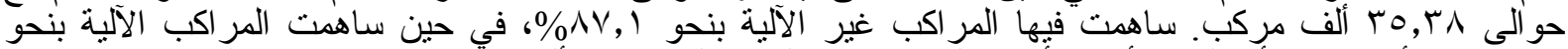

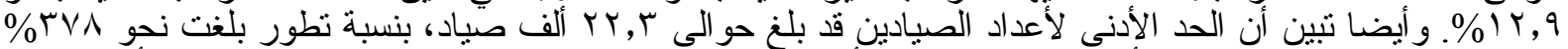

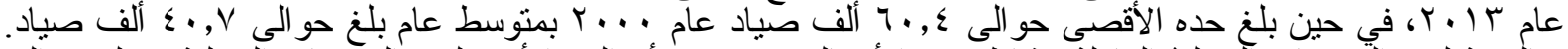

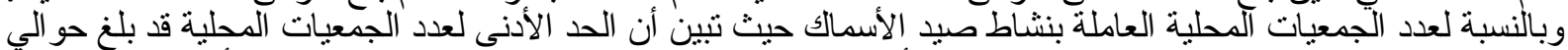

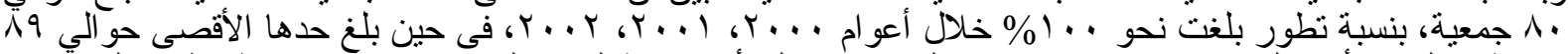

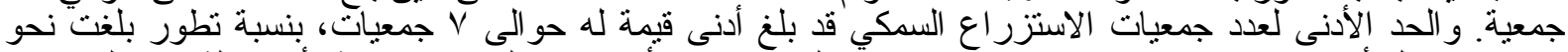

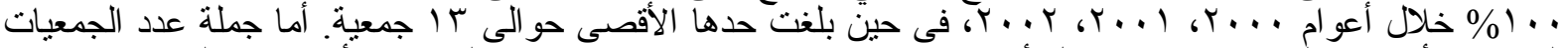

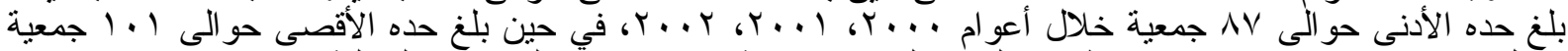

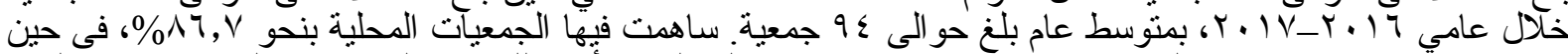

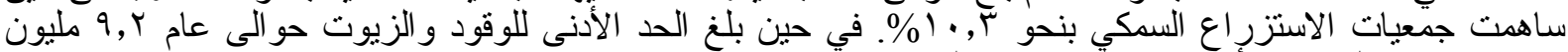

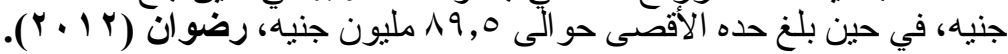

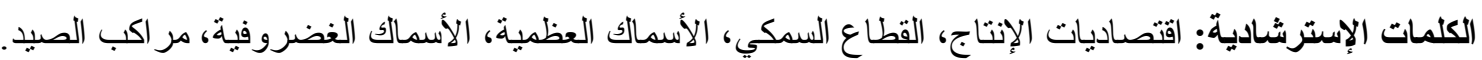


